AIAA-2002-1513

\title{
BUCKLING LOAD CALCULATIONS OF THE ISOTROPIC SHELL A-8 USING A HIGH-FIDELITY HIERARCHICAL APPROACH
}

\author{
Johann Arbocz* \\ Delft University of Technology, The Netherlands \\ and \\ James H. Starnes** \\ NASA Langley Research Center, Hampton, VA 23681-001
}

\begin{abstract}
As a step towards developing a new design philosophy, one that moves away from the traditional empirical approach used today in design towards a science-based design technology approach, a test series of 7 isotropic shells carried out by Arbocz and Babcock [1] at Caltech is used. It is shown how the hierarchical approach to buckling load calculations proposed by Arbocz et al [2] can be used to perform an approach often called "high fidelity analysis", where the uncertainties involved in a design are simulated by refined and accurate numerical methods. The Delft Interactive Shell DEsign COde (short, DISDECO) is employed for this hierarchical analysis to provide an accurate prediction of the critical buckling load of the given shell structure. This value is used later as a reference to establish the accuracy of the Level-3 buckling load predictions. As a final step in the hierarchical analysis approach, the critical buckling load and the estimated imperfection sensitivity of the shell are verified by conducting an analysis using a sutficiently refined finite element model with one of the current generation two-dimensional shell analysis codes with the advanced capabilities needed to represent both geometric and material nonlinearities.
\end{abstract}

\footnotetext{
* Professor, Faculty of Aerospace Engineering, Fellow AIAA

* Senior Engineer, Structures and Materials Competency, Fellow AIAA

Copyright $\odot 2002$ by J. Arbocz and J.H. Starnes

Published by AIAA with permission
}

\section{INTODUCTION}

It is generally agreed that, in order to make the development of the Advanced Space Transportation System a success and to achieve the very ambitious performance goals (like every generation of vehicles $10 \times$ safer and $10 x$ cheaper than the previous one), one must make full and efficient use of the technical expertise accumulated in the past $\mathbf{5 0}$ years or so, and combine it with the tremendous computational power now available. It is obvious that with the strict weight constraints used in space applications these performance goals can only be achieved with an approach often called "high fidelity analysis", where the uncertainties involved in a design are simulated by refined and accurate numerical models. In the end the use of "high fidelity" numerical simulation will also lead to overall cost reduction, since the analysis and design phase will be completed faster and only the reliability of the final configuration needs to be verified by structural testing.

The light-weight shell structures used in aerospace applications are often buckling critical. The buckling load calculations are usually carried out by one of the many currently available finite element based computer codes [e.g., 3,4]. In order to reduce computer execution time, buckling analyses are often done using only the small displacement stiffness matrix $K_{0}$. This approach is used, despite the fact that the "initial stability problem" so formulated can only give physically meaningful answers if the elastic solutions based on $K_{0}$ (at least approximately) are identically equal to zero [5].

When the qualitative nature of the expected behavior is completely unknown, the stability of the structure must be investigated using the full tangent stiffness matrix $\mathrm{K}_{\mathrm{T}}$ in order to guarantee accurate and reliable buckling load and buckling mode predictions. In order to discover the load level at which $K_{T}$ ceases to be positive definite (that is, the load level when 
buckling occurs), a step-by-step analysis procedure is needed.

In addition, it is imperative (though often completely neglected), that at the beginning of any stability investigation, the accuracy of the discrete model used should be checked against available analytical or semi-analytical results. This step is part of a mandatory study needed in order to establish the dependence of the buckling load predictions on the mesh distribution used. Furthermore, as has been pointed out in the past by Byskov [6], if one carries out imperfection sensitivity investigations, which involve an extension of the solution into the postbuckling response region, further mesh refinement may be needed since the wavelength of the dominant large deformation pattern may often decrease significantly.

Finally, whenever one is engaged in shell stability analysis it is especially important that one is aware of the possible detrimental effects of a whole series of factors, that have been investigated extensively in the late 1960 s and the early 1970s. Thus for an accurate and reliable prediction of the critical buckling load of a real structure, one must account not only for the influence of initial imperfections [e.g., 7,8 ] and of the boundary conditions [e.g. 9], but one must also consider the effects of stiffener and load eccentricity [e.g., 10] and the prebuckling deformations caused by the edge restraints [e.g. 11,12].

A test series of 7 isotropic shells carried out by Arbocz and Babcock [1] at Caltech is used to illustrate how such a hierarchical approach to buckling load calculations can be carried out. The platform for the multi-level computations, needed for an accurate prediction of the critical buckling loads and a reliable estimation of their imperfection sensitivity, is provided by DISDECO [13]. With this open ended, hierarchical, interactive computer code the user can access from his workstation a succession of programs of increasing complexity.

\section{SOLUTION OF THE BUCKLING PROBLEM}

In the following it will be shown that with the help of DISDECO, the Delft Interactive Shell DEsign COde, the shell designer can study the buckling behavior of a specified shell, calculate its critical buckling load quite accurately and make a reliable prediction of the expected degree of imperfection sensitivity of the critical buckling load. The proposed procedure consists of a hierarchical approach, where the analyst proceeds step-by-step from the simpler (Level-1) methods used by the early investigators to the more sophisticated analytical and numerical (Level-2 and Level-3) methods used presently.

\section{Level-1 Perfect Shell Buckling Analysis}

The geometric and material properties of the isotropic shell $A-8$ of Ref. [1] are listed in Table 1.

Table 1.

Geometric properties of Caltech isotropic shell A-8

\begin{tabular}{rllll|}
$t_{\text {total }}(=h)$ & $=0.00464$ & in $(=0.117856$ & $\mathrm{mm})$ \\
$L$ & $=8.0$ & in $(=203.2$ & $\mathrm{mm})$ \\
$R$ & $=4.0$ & in $(=101.6$ & $\mathrm{mm}$ & ) \\
$E$ & $=15.2 \times 10^{6}$ & psi $\left(=1.0480 \times 10^{5}\right.$ & $\left.\mathrm{N} / \mathrm{mm}^{2}\right)$ \\
$v$ & $=0.3$ & & & \\
\hline
\end{tabular}

Assuming a perfect shell $(\bar{W}=0)$ and the following membrane prebuckling state

$$
\begin{aligned}
& W^{(0)}=h W_{v}=h \bar{A}_{12}^{*} \frac{\lambda}{c} \\
& F^{(0)}=-\frac{E h^{2}}{c R} \frac{1}{2} \lambda y^{2}
\end{aligned}
$$

where

$$
\begin{aligned}
& \lambda=\frac{\sigma}{\sigma_{c \ell}}=\frac{N_{x}}{N_{c \ell}} ; \sigma_{c \ell}=\frac{E h}{c R} ; N_{c \ell}=\sigma_{c \ell} h \\
& \text { and } c=\sqrt{3\left(1-v^{2}\right)}
\end{aligned}
$$

then the nonlinear equations governing the prebuckling state are identically satisfied and the linearlzed stability equations reduce to a set of equations with constant coefficients. It has been shown in Ref. [14] that by assuming an asymmetric bifurcation mode of the form

$$
W^{(1)}=h \sin m \pi \frac{x}{L} \cos n \frac{y}{R}
$$

where

$$
\begin{aligned}
& \mathrm{m}=\mathrm{k}=\text { number of axial half waves } \\
& \mathrm{n}=\ell=\text { number of circumferential full waves }
\end{aligned}
$$

one can reduce the solution of the linearized stability equations to an algebraic eigenvalue problem. Notice that the eigenvalue $\lambda_{\mathrm{mn}}$ depends on the wave numbers $m$ and $n$. The critical load parameter $\lambda_{c}$ is the lowest of all possible eigenvalues. Thus finding $\lambda_{c}$ involves a search over the integer valued wave numbers $m$ and $n$. Using the Level-1 
computational module AXBIF [14] a search over integer valued axial half-wave numbers $m$ yielded the lowest eigenvalues listed in Table 2 for the specitied circumferential wave numbers n.

Table 2.

Buckling loads of the Caltech isotropic shell A-8 Buckling load map for the perfect shell using AXBIF [14] $\left(\mathrm{N}_{\mathrm{C} \ell}=-49.51517 \mathrm{lb} / \mathrm{in}\right)$

\begin{tabular}{|lll|}
$n=4$ & $\lambda_{c}^{m}=1.000$ & $(m=34)$ \\
$n=5$ & $\lambda_{c}^{m}=1.000$ & $(m=34)$ \\
$n=6$ & $\lambda_{c}^{m}=1.000$ & $(m=34)$ \\
$n=7$ & $\lambda_{c}^{m}=1.000$ & $(m=33)$ \\
$n=8$ & $\lambda_{c}^{m}=1.000$ & $(m=33)$ \\
$n=9$ & $\lambda_{c}^{m}=1.000$ & $(m=1)$ \\
& $\lambda_{c}^{m}=1.000$ & $(m=33)$ \\
$n=10$ & $\lambda_{c}^{m}=1.000$ & $(m=33)$ \\
$n=11$ & $\lambda_{c}^{m}=1.000$ & $(m=33)$ \\
$n=12$ & $\lambda_{c}^{m}=1.000$ & $(m=32)$ \\
$n=13$ & $\lambda_{c}^{m}=1.008$ & $(m=2)$ \\
& $\lambda_{c}^{m}=1.000$ & $(m=32)$ \\
$n=14$ & $\lambda_{c}^{m}=1.000$ & $(m=32)$ \\
$n=15$ & $\lambda_{c}^{m}=1.001$ & $(m=3)$ \\
& $\lambda_{c}^{m}=1.000$ & $(m=31)$ \\
\hline
\end{tabular}

As is known the lowest eigenvalue is a repeated eigenvalue with a high degree of multiplicity. The family of modes belonging to the lowest eigenvalue $\lambda_{c}=1.000$ lie on the socalled Koiter circle [7].

To facilitate the interpretation of the numerical results obtained, DISDECO provides the user with various graphical interfaces.

Thus the results of the search for the critical (lowest) buckling load $\lambda_{c}$ can be displayed in a contour map as shown in Fig. 1. In order to provide a quick overview of the distribution of eigenvalues, the values displayed in the contour plot are nomalized. Thus in Fig. 1 the following normalized eigenvalues are plotted

$$
\lambda_{\mathrm{c}}^{\mathrm{m}}=\frac{\mathrm{N}_{\mathrm{c}}^{\mathrm{m}}}{-49.51517}
$$

Notice that the critical buckling load can be calculated using a simple multiplication

$$
\begin{aligned}
& \mathrm{N}_{c}^{m}=\lambda_{c}^{m} \mathrm{~N}_{c \ell}= \\
& 1.000(-49.51517)=-49.51517 \mathrm{lb} / \mathrm{in}
\end{aligned}
$$

\section{Level-2 Perfect Shell Buckling Analysis}

To investigate the effects of edge constraint and of different boundary conditions on the critical buckling load of the perfect shell $(\bar{W}=0)$ one has to switch to the Level-2 computational module ANILISA [15]. In this module the axisymmetric prebuckling state is represented by

$$
\begin{aligned}
& W^{(0)}=h W_{v}+h w_{0}(x) \\
& F^{(0)}=\frac{E h^{2}}{c R}\left[-\frac{1}{2} \lambda y^{2}+R^{2} f_{0}(x)\right]
\end{aligned}
$$

It has been shown in Ref. [15] that with these assumptions the prebuckling problem is reduced to the solution of a single fourth order ordinary differential equation with constant coefficients, which always admits exponential solutions. Closed form solutions for simply supported and clamped boundary conditions have been published in the literature [16].

For isotropic shells the linearized stability equations admit separable solutions of the form

$$
\begin{aligned}
& W^{(1)}=h w_{1}(x) \cos n \theta \\
& F^{(1)}=\frac{E R h^{2}}{c} f_{1}(x) \cos n \theta
\end{aligned}
$$

where $\theta=\frac{y}{R}$.

Using a generalization of Stodola's method [17] first published by Cohen [18] the resulting nonlinear eigenvalue problem is reduced to a sequence of linearized eigenvalue problems. The resulting ordinary differential equations are solved numerically by a technique known as "parallel shooting over $\mathrm{N}$-intervals" [19]. Notice that by this approach the effect of edge restraint and the specific boundary conditions are satisfied rigorously. To find the critical load parameter $\lambda_{C}$ an n-search must be carried out, whereby one must be careful to find not a local minimum but the absolute minimum. As can be seen from the results presented in Table 3 the n-search using membrane prebuckling and a rigorous satisfaction of SS-3 $\left(N_{X}=v=w=M_{X}=0\right.$ ) boundary conditions for the stability problem yields indeed the repeated eigenvalues $\lambda_{c}^{m}-1.0$ located on the "Koiter" 
circle. The slight variations in the eigenvalues are due to the fact that both the axial half-wave numbers $m$ and the circumferential full wave numbers $n$ are integers.

Table 3

Buckling loads of the Caltech isotropic shell A-8 $\left(\mathrm{N}_{\mathrm{c} \ell}=-49.51517 \mathrm{lb} / \mathrm{in}\right)$

Buckling load map for the perfect shell using ANILISA [15] (B.C. $N_{x}=v=w=M_{x}=0$ )

\begin{tabular}{|c|c|c|}
\hline Prebuckling: & Membrane & Nonlinear \\
\hline \multirow[t]{2}{*}{$n=8$} & $\lambda_{c}^{m}=1.10977(m=1)$ & $\lambda_{c}^{n l}=0.951155$ \\
\hline & $\lambda_{c}^{m}=1.00001(m=33)$ & $\lambda_{c}^{n l}=0.966181^{*}$ \\
\hline \multirow[t]{2}{*}{$n=9$} & $\lambda_{c}^{m}=1.00004(m=1)$ & $\lambda_{c}^{n \prime}=0.934990$ \\
\hline & $\lambda_{c}^{m}=1.00000(m=33)$ & $\lambda_{c}^{\mathrm{nl}}=0.957035^{*}$ \\
\hline \multirow[t]{2}{*}{$n=10$} & $\lambda_{c}^{m}=1.08160(m=1)$ & $\lambda_{c}^{\mathrm{nl}}=0.940209$ \\
\hline & $\lambda_{c}^{m}=1.00011(m=33)$ & $\lambda_{c}^{\mathrm{nl}}=0.946124^{*}$ \\
\hline \multirow[t]{2}{*}{$n=11$} & $\lambda_{c}^{m}=1.12539(m=2)$ & $\lambda_{c}^{n l}=0.931492^{*}$ \\
\hline & $\lambda_{c}^{m}=1.00035(m=32)$ & $\lambda_{c}^{n l}=0.939878$ \\
\hline \multirow[t]{2}{*}{$n=12$} & $\lambda_{c}^{m}=1.01481(m=2)$ & $\lambda_{c}^{n l}=0.915200^{*}$ \\
\hline & $\lambda_{c}^{m}=1.00004(m=32)$ & $\lambda_{c}^{n I}=0.931464$ \\
\hline \multirow[t]{2}{*}{$n=13$} & $\lambda_{c}^{m}=1.00836(m=2)$ & $\lambda_{c}^{\mathrm{nl}}=0.908528^{\star}$ \\
\hline & $\lambda_{c}^{m}=1.00004(m=32)$ & $\lambda_{c}^{n l}=0.918482$ \\
\hline \multirow[t]{2}{*}{$n=14$} & $\lambda_{c}^{m}=1.04064(m=3)$ & $\lambda_{c}^{n l}=0.903256$ \\
\hline & $\lambda_{c}^{m}=1.00030(m=31)$ & $\lambda_{c}^{n l}=0.906963^{*}$ \\
\hline \multirow[t]{2}{*}{$n=15$} & $\lambda_{c}^{m}=1.00060(m=3)$ & $\lambda_{c}^{n l}=0.891368$ \\
\hline & $\lambda_{c}^{m}=1.00000(m=31)$ & $\lambda_{c}^{n l}=0.898837^{*}$ \\
\hline \multirow[t]{2}{*}{$n=16$} & $\lambda_{c}^{m}=1.02041(m=3)$ & $\lambda_{c}^{n l}=0.885185$ \\
\hline & $\lambda_{c}^{m}=1.00023(m=31)$ & $\lambda_{c}^{n \mathrm{n}}=0.886606^{*}$ \\
\hline \multirow[t]{2}{*}{$n=17$} & $\lambda_{c}^{m}=1.00086(m=4)$ & $\lambda_{c}^{n l}=0.875881^{*}$ \\
\hline & $\lambda_{c}^{m}=1.00001(m=30)$ & $\lambda_{c}^{n \mathrm{n}}=0.878312$ \\
\hline \multirow[t]{2}{*}{$n=18$} & $\lambda_{c}^{m}=1.01300(m=4)$ & $\lambda_{c}^{n l}=0.868406^{*}$ \\
\hline & $\lambda_{c}^{m}=1.00027(m=30)$ & $\lambda_{c}^{n l}=0.869200$ \\
\hline \multirow[t]{2}{*}{$n=19$} & $\lambda_{c}^{m}=1.00014(m=5)$ & $\lambda_{c}^{n l}=0.861087$ \\
\hline & $\lambda_{c}^{m}=1.00001(m=29)$ & $\lambda_{c}^{n l}=0.861626^{*}$ \\
\hline \multirow[t]{2}{*}{$n=20$} & $\lambda_{c}^{m}=1.00164(m=6)$ & $\lambda_{c}^{n \mathrm{l}}=0.854891$ \\
\hline & $\lambda_{c}^{m}=1.00006(m=28)$ & $\lambda_{c}^{n l}=0.855033^{*}$ \\
\hline
\end{tabular}

Table 3 - continuation

\begin{tabular}{|lll|}
\hline Prebuckling: & Membrane & Nonlinear \\
$n=21$ & $\lambda_{c}^{m}=1.00378(m=7)$ & $\lambda_{c}^{n l}=0.849839^{*}$ \\
& $\lambda_{c}^{m}=1.00023(m=27)$ & $\lambda_{c}^{n l}=0.849927$ \\
$n=22$ & $\lambda_{c}^{m}=1.00183(m=7)$ & $\lambda_{c}^{n l}=0.846336^{*}$ \\
& $\lambda_{c}^{m}=1.00014(m=27)$ & $\lambda_{c}^{n l}=0.846344$ \\
$n=23$ & $\lambda_{c}^{m}=1.00114(m=8)$ & $\lambda_{c}^{n l}=0.844502$ \\
& $\lambda_{c}^{m}=1.00012(m=26)$ & $\lambda_{c}^{n l}=0.844511^{*}$ \\
$n=24$ & $\lambda_{c}^{m}=1.00071(m=10)$ & $\lambda_{c}^{n l}=0.844480^{*}$ \\
& $\lambda_{c}^{m}=1.00011(m=24)$ & $\lambda_{c}^{n l}=0.844481$ \\
$n=25$ & $\lambda_{c}^{m}=1.00000(m=11)$ & $\lambda_{c}^{n l}=0.846279^{*}$ \\
& $\lambda_{c}^{m}=1.00000(m=23)$ & $\lambda_{c}^{n l}=0.846280$ \\
$n=26$ & $\lambda_{c}^{m}=1.00002(m=13)$ & $\lambda_{c}^{n l}=0.849833^{*}$ \\
& $\lambda_{c}^{m}=1.00001(m=21)$ & $\lambda_{c}^{n l}=0.849833$ \\
$n=27$ & $\lambda_{c}^{m}=1.00027(m=17)$ & $\lambda_{c}^{n l}=0.854990^{*}$ \\
& $\lambda_{c}^{m}=1.00032(m=18)$ & $\lambda_{c}^{n l}=0.854990$ \\
\end{tabular}

* anti-sym at $\mathrm{x}=\mathrm{L} / 2$.

The most accurate Level-2 solutions are obtained when one employs a rigorous nonlinear prebuckling analysis. As can be seen from the results listed in Table 3 , for this particular shell the critical buckling loads with nonlinear prebuckling are always lower than the corresponding results obtained using a membrane prebuckling analysis. Notice that the critical load $N_{C}$ can be calculated easily by multiplying the lowest eigenvalue $\lambda_{c}$ by the normalizing factor $\mathrm{N}_{\mathrm{c} \ell}=-49.51517 \mathrm{lb} / \mathrm{in}$ yielding

$$
N_{c}=\lambda_{c} N_{c \ell}=-41.815 \mathrm{lb} / \mathrm{in} \quad(n=24)
$$

In fig. 2 the critical buckling modes using membrane and rigorous nonlinear prebuckling are depicted. Notice that the solutions with nonlinear prebuckling differ significantly from the ones obtained using membrane prebuckling, especially at $n=24$ where one observes a typical edge buckling type behavior.

\section{Level-3 Perfect Shell Buckling Analygis}

To verify the earlier predictions the finite difference version [20] of the well known shell 
analysis code STAGS [21] will be used. In order to be able to represent the measured initial imperfections accurately, the whole shell will be modeled.

Initially a convergence study must be carried out in order to establish the mesh size needed for accurate modeling of the buckling behavior of the shell in question. For this purpose the asymmetric bifurcation from a nonlinear prebuckling path option was used, whereby the earlier results obtained with the Level-2 module ANILISA listed in Table 3 serve as a reference.

In the convergence study, at first, for a fixed number of mesh points in the axial direction (NR $=161$ ) the number of mesh points in the circumferential direction (NC) was increased until the bifurcation load approached a horizontal tangent. As can be seen from Fig. 3 the results converge to a limiting value from below at about $N C=261$. Next, for a fixed number of mesh points in the circumferential direction (NC = 161) the number of rows (NA) was varied. This time convergence is from above and as can be seen from Fig. 3 the horizontal tangent is reached at about NR = 261.

To illustrate the difference between using coarser meshes to speed up the computations and the finer meshes which produce (nearly) converged solutions, initially the first three eigenvalues and eigenvectors are computed.

Using a mesh of 161 rows and 181 columns (a model with 89488 D.O.F.'s and a maximum semi-bandwidth of 713) and SS-3 boundary conditions $\left(N_{x}=-N_{0}, v=W=M_{X}=0\right)$ the following 3 lowest eigenvalues were obtained:

$$
\begin{gathered}
\lambda_{C}^{(1)}=0.833361 \quad(n=26) \quad \rightarrow \\
N_{C}^{(1)}=\lambda_{C}^{(1)} N_{C \ell}=-41.264 \mathrm{lb} / \mathrm{in} \\
\lambda_{C}^{(2)}=0.833374 \quad(n=26) \quad \rightarrow \\
N_{C}^{(2)}=\lambda_{C}^{(2)} N_{C \ell}=-41.265 \mathrm{lb} / \mathrm{in} \\
\lambda_{C}^{(3)}=0.834238 \quad(n=25) \quad \rightarrow \\
N_{C}^{(3)}=\lambda_{C}^{(3)} N_{C \ell}=-41.307 \mathrm{lb} / \mathrm{in}
\end{gathered}
$$

Next these computations were repeated using a mesh of 261 rows and 261 columns (a model with 207508 D.O.F.'s and a maximum semibandwidth of 1037) and the same SS-3 boundary conditions yielding the following results

$$
\begin{gathered}
\lambda_{C}^{(1)}=0.844993 \quad(n=24) \quad \rightarrow \\
N_{C}^{(1)}=\lambda_{C}^{(1)} N_{C \ell}=-41.840 \mathrm{lb} / \mathrm{in} \\
\lambda_{C}^{(2)}=0.845006 \quad(n=24) \quad \rightarrow \\
N_{C}^{(2)}=\lambda_{C}^{(2)} N_{C \ell}=-41.841 \mathrm{lb} / \mathrm{in} \\
\lambda_{C}^{(3)}=0.845100 \quad(n=25) \quad \rightarrow \\
N_{C}^{(3)}=\lambda_{C}^{(3)} N_{C \ell}=-41.845 \mathrm{lb} / \mathrm{in}
\end{gathered}
$$

Details of the critical mode are displayed in Figures 4-5 for the coarser $161 \times 181$ model and in Figures 6-7 for the finer 261-261 model. Notice that using the more refined $261 \times 261$ model the sequence of the 3 lowest buckling loads and the corresponding buckling modes agree closely with the predictions obtained with the Level-2 module ANILISA (see also Table 3)

\section{IMPERFECTION SENSITIVITY STUDY}

That initial imperfections may decrease the load carrying capacity of thin-walled shell structures is by now widely known and accepted. However, in order to calculate the effect of initial imperfections one must know their shape and amplitude, an information that is rarely available.

In the absence of initial imperfection measurements, as a first step one must establish whether a given shell-loading combination is imperfection sensitive, and if the answer is positive to estimate how damaging certain characteristic imperfection shapes are.

\section{Single Axisymmetric Imperfection}

Based on Koiter's pioneering work on the effect of initial imperfections $[7,22]$ the simplest imperfection model consists of a single axisymmetric imperfection

$$
\bar{W}=h \bar{\xi}_{1} \cos i \pi \frac{x}{L}
$$

where $i$ is an integer denoting the number of half-waves in the axial direction and $\bar{\xi}_{1}$ is the amplitude of the axisymmetric imperfection normalized by the shell wall-thickness $h$.

If one assumes that both the axial load and the boundary conditions are independent of the circumferential coordinate, then the prebuck- 
ling solution will also be axisymmetric, a fact that simplifies the solution considerably.

\section{Level-1 Analysis of Axisymmetric} Imperfection

Neglecting the effect of the prebuckling boundary conditions the nonilinear equations governing the prebuckling state admit the following axisymmetric solutions

$$
\begin{aligned}
& W^{(0)}=h W_{v}+h W_{o}(x) \\
& F^{(0)}=-\frac{E h^{2}}{c R} \frac{1}{2} \lambda y^{2}+f_{o}(x)
\end{aligned}
$$

where

$$
\begin{gathered}
w_{o}(x)=\frac{\lambda}{\lambda_{c_{i}}-\lambda} h \bar{\xi}_{1} \cos i \pi \frac{x}{L} \\
f_{o}(x)=\frac{\lambda}{\lambda_{c_{i}}-\lambda} \frac{\left(1+\bar{B}_{\left.21 \alpha_{i}^{2}\right)}^{2} \frac{E h^{3}}{2 \alpha_{i}^{2} \bar{A}_{22}^{*}} \bar{\xi}_{1} \cos i \pi \frac{x}{L}\right.}{c} \\
\lambda_{c_{i}}=\frac{1}{2}\left\{\alpha_{i}^{2} \bar{D}_{11}^{\star}+\frac{\left(1+\bar{B}_{21 \alpha_{j}^{2}}^{2}\right)^{2}}{2 \alpha_{i}^{2} \bar{A}_{22}^{\star}}\right\}
\end{gathered}
$$

Notice that the linearized stability equations become now a set of equations with variable coefficients. The reduced wave number $\boldsymbol{\alpha}_{\mathbf{j}}$ and the normalized stiffness coefficients $\bar{A}_{22}^{*}$, $\bar{B}_{21}$ and $\bar{D}_{11}^{*}$ are all listed in Ref. [14].

It has been shown in Ref. [14] that by assuming an asymmetric bifurcation mode of the form

$$
w^{(1)}=h \sin m \pi \frac{x}{L} \cos n \frac{y}{R}
$$

where

$$
\begin{aligned}
& m=k=\text { number of axial half waves } \\
& n=\ell=\text { number of circumferential full waves }
\end{aligned}
$$

a Galerkin type approximate solution yields for the eigenvalues (read, buckling loads) $\lambda$ of the problem a characteristic equation in the form of a cubic polynomial

$$
\begin{aligned}
& \lambda^{3}-\left(\lambda_{m n \tau}+2 \lambda_{c_{i}}-\hat{C}_{1} \bar{\xi}_{1} \delta_{i=2 m}\right) \lambda^{2} \\
& +\left\{2 \lambda_{m n \tau}+\lambda_{c_{i}}+\left(\hat{C}_{2}-\hat{C}_{1}\right) \bar{\xi}_{1} \delta_{i=2 m}\right\} \lambda_{c_{i}} \lambda \\
& -\left\{\lambda_{m n \tau}+\hat{C}_{2} \bar{\xi}_{1} \delta_{i=2 m}+\left(\hat{C}_{3}-\hat{C}_{4} \delta_{i m}\right) \bar{\xi}_{1}^{2}\right\} \lambda_{c_{i}}^{2}=0
\end{aligned}
$$

where

$$
\begin{aligned}
\begin{aligned}
\delta_{i=2 m}=1 & \text { if } i=2 m \\
=0 & \text { otherwise }
\end{aligned} \\
\begin{aligned}
\delta_{i m}=1 & \text { if } \mathrm{i}=\mathrm{m} \\
=0 & \text { otherwise }
\end{aligned}
\end{aligned}
$$

and the constants $\hat{\mathrm{C}}_{1}, \hat{\mathrm{C}}_{2}, \ldots$ are listed in Appendix C of Ref. [14].

Here it must be remembered that one will only get any noticeable degrading influence of the assumed axisymmetric imperfection if $\bar{\xi}_{1}$ is negative and if the coupling condition $\mathrm{i}=2 \mathrm{~m}$ is satisfied. The physical explanation for this can be found in Kolter's 1963 paper [22]. Furthermore, in order to obtain the smallest real root of Eq. (9), for a given axisymmetric imperfection $\bar{\xi}_{1}$ an n-search must be carried out. It should also be noticed that the terms involving the Kronecker delta $\delta_{i=2 m}$ are all linear in $\bar{\xi}_{1}$, and thus they dominate the buckling behavior of the shell with axisymmetric imperfection.

Assuming that the most likely axisymmetric imperfection of the silver pointed wax mandrel used to electroplate the isotropic shell A-8 is given by

$$
\bar{W}=h \bar{\xi}_{1} \cos 2 \pi \frac{x}{L}
$$

the Level-1 DISDECO computational module AXBIF generated the solid curve shown in Fig. 8. Notice that the curve is normalized by $N_{c \ell}=-49.51517 \mathrm{lb} / \mathrm{in}$, the classical buckling load of an isotropic shell, obtained around 1910 by Lorenz [23], Timoshenko [24] and Southwell [25]. Notice also that an initial imperfection amplitude equal to the wall thickness of the shell $\left(\bar{\xi}_{1}=-1.0\right)$ generates a "knockdown factor" of $\lambda_{c}=0.706$, resulting in the following buckling load

$$
\begin{aligned}
N_{c}= & \lambda_{c} N_{c \ell}= \\
& 0.706(-49.51517)=-34.958 \mathrm{lb} / \mathrm{in}
\end{aligned}
$$

However, if one assumes an imperfection in the form of the classic axisymmetric buckling mode of Koiter [7]

$$
\bar{W}=h \bar{\xi}_{1} \cos i_{c \ell} \pi \frac{x}{L}
$$

where 


$$
i_{C \ell}=\frac{L}{\pi} \sqrt{\frac{2 c}{R h}} ; c=\sqrt{3\left(1-v^{2}\right)}
$$

then for the isotropic shell $A-8 i_{c \ell}-34.0$, and the Level-1 DISDECO computational module AXBIF found that the minimum buckling load occurred when $m=\frac{1}{2} i_{c \ell}$ for different values of $n$ (which depended on the value of $\xi_{1}$ ). These results are shown in Fig. 9.

For this case an initial imperfection amplitude equal to the wall thickness of the shell $\left(\xi_{1}=-1.0\right)$ generates a "knockdown factor" of $\lambda_{c}=0.196$ resulting in the following rather low buckling load

$$
\begin{aligned}
& N_{c}=\lambda_{c} N_{c \ell}= \\
& \quad 0.196(-49.51517)=-9.705 \mathrm{lb} / \mathrm{in}
\end{aligned}
$$

The last two buckling loads clearly demonstrate the importance of the shape of the initial imperfections involved and the role of the corresponding eigenvalues. Using the long wave axisymmetric imperfection $(i=2)$ the corresponding normalized axisymmetric buckling load (eigenvalue) of the perfect shell is $\lambda_{c_{i}}=144.321$ (a very high value) yielding a low imperfection sensitivity. For the short wave imperfection $(i=34)$ the corresponding normalized axisymmetric buckling load (eigenvalue) of the perfect shell is $\lambda_{c_{i}}=1.000$. Thus it lies on Koiter's circle yielding a high imperfection sensitivity.

\section{Level-2 Analysis of Axisymmetric} imperfection

Since the external loading, the boundary conditions and the assumed initial imperfection are axisymmetric, therefore the prebuckling solution will also be axisymmetric. It has been shown in Ref. [26] that by assuming

$$
\begin{aligned}
& W^{(0)}=h W v+h W_{0}(x) \\
& F^{(0)}=\frac{E h^{2}}{c R}\left\{-\frac{1}{2} \lambda y^{2}+R^{2} f_{O}(x)\right\}
\end{aligned}
$$

the solution of the nonlinear partial differential equations governing the prebuckling state can be reduced to the solution of a single fourth order ordinary differential equation with constant coefficients, which can be solved routinely.

For isotropic shells the resulting linearized stability equations admit separable solutions of the form

$$
\begin{aligned}
& W^{(1)}=h W_{1}(x) \cos n \theta \\
& F^{(1)}=\frac{E R h^{2}}{c} f_{1}(x) \cos n \theta
\end{aligned}
$$

where $\theta=\frac{y}{R}$.

Solution proceeds as outlined on Ref. [26]. Using an updated version of the Level-2 computational module MANILISA [27] and SS$3\left(N_{X}=v=W=M_{X}=0\right)$ boundary conditions one obtains the results presented in Table 4. Notice that a rigorous nonlinear prebuckling analysis was used and an n-search was carried out for each specified axisymmetric imperfection amplitude $\vec{\xi}_{1}$.

The values of Table 4 are plotted as the dashed curve in Fig. 9. A comparison of the results obtained via the Level-1 module AXBIF (solid curve) and the Level-2 module MANILISA (dashed curve) shows that also in the case of axisymmetric imperfections a rigorous pre-buckling analysis should be used. Especially for very small initial imperfection amplitudes $\left(\left|F_{1}\right|<0.1\right)$ the Level-1 predictions are inaccurate and overestimate the critical buckling load. Notice further that both curves have been normalized by classical buckling load $N_{c \ell}=-49.51517 \mathrm{lb} / \mathrm{in}$. This way the effect of using a rigorous prebuckling analysis becomes easily discernible.

Table 4

Buckling loads of the Caltech isotropic shell A-8 $\left(\mathrm{N}_{\mathrm{c} \ell}=-49.51517 \mathrm{lb} / \mathrm{in}\right)$

Short wave axisymmetric imperfection $\bar{W} / \mathrm{h}=\bar{\xi}_{1} \cos 34 \pi \frac{\mathrm{x}}{\mathrm{L}}$ using MANILISA [27]

(B.C. $: N_{X}=v=w=M_{X}=0$ )

\begin{tabular}{|l|l|l|l|}
\hline $\bar{\xi}_{1}$ & $\lambda_{c}^{n l}$ & $\bar{\xi}_{1}$ & $\lambda_{c}^{n l}$ \\
\hline 0. & $0.844480(n=24)$ & -0.1 & $0.549168(n=17)$ \\
-0.01 & $0.847663(n=21)$ & -0.2 & $0.420091(n=17)$ \\
-0.02 & $0.786079(n=21)$ & -0.3 & $0.354645(n=19)$ \\
-0.03 & $0.740449(n=21)$ & -0.4 & $0.305484(n=18)$ \\
-0.04 & $0.703421(n=21)$ & -0.5 & $0.271507(n=17)$ \\
-0.05 & $0.671958(n=21)$ & -0.6 & $0.247002(n=16)$ \\
-0.06 & $0.644481(n=21)$ & -0.7 & $0.229213(n=15)$ \\
-0.07 & $0.620047(n=21)$ & -0.8 & $0.214064(n=20)$ \\
-0.08 & $0.598039(n=21)$ & -0.9 & $0.200103(n=19)$ \\
-0.09 & $0.578030(n=21)$ & -1.0 & $0.189011(n=18)$ \\
\hline
\end{tabular}


Level-3 Analysis of Axisymmetric Imperfection

Recalling that since both the axial load and the boundary conditions are independent of the circumferential coordinate, therefore the prebuckling solution will also be axisymmetric, one can use once again the asymmetric bifurcation from a nonlinear prebuckling path option. By modeling the full shell the code can choose itself the critical number of full waves in the circumferential direction. No n-search must be carried out. Using a uniformly spaced mesh of 161 rows and 181 columns and the user written subroutine option WIMP to introduce the following axisymmetric imperfection

$$
\bar{W}=10 h \cos 2 \pi \frac{x}{L}
$$

(remember STAGS defines $\bar{W}$ positive outward) the following critical bifurcation load was found

$$
N_{C}=-32.582 \mathrm{lb} / \mathrm{in}
$$

As can be seen from Fig. 10 the critical buckling mode has $n=9$ full waves in the circumferential direction and a single half wave in the axial direction. The nondimensional bifurcation load of the shell with axisymmetric imperfection is for $\bar{\xi}_{1}=1.0$

$$
\lambda_{c}=\frac{N_{C}}{N_{c \ell}}=\frac{-32.582}{-49.51517}=0.65802
$$

Notice also that the Level-2 MANILISA prediction $\left(\lambda_{C}^{n l}=0.683, n=9\right)$ agrees closely with the Level-3 STAGS-A prediction $\left(\lambda_{c}^{n l}=0.658, n=9\right)$. The slight difference is partly due to the fact that MANILISA uses the Donnell type nonlinear shell equations, whereas STAGS-A employs the higher order Marlowe-Flügge equations.

\section{Single Asymmetric Imperfection}

The effect of a single asymmetric initial imperfection can be investigated either by solving the full nonlinear response problem or by employing the well known LyapunovSchmidt-Koiter [7] reduction technique. When investigating the degrading effect of a single mode asymmetric imperfection

$$
\bar{W}=t \bar{\xi}_{2} \sin m \pi \frac{x}{L} \cos n \frac{y}{R}
$$

where $m$ and $n$ are integers denoting the number of axial half-waves and the number of circumferential full waves, respectively, instability occurs at the limit point of the prebuckling state in the generalized loaddeformation space. Assuming that the eigenvalue problem for the critical (lowest) buckling load $\Lambda_{c}$ will yield a unique asym-

metric buckling mode $W^{(1)}$, then for an imperfect shell $\left(\bar{\xi}_{2} \neq 0\right)$ the shape of the generalized load-deflection curve in the vicinity of the bifurcation point $\Lambda=\Lambda_{c}$ is given by the following asymptotic expansion

$$
\begin{aligned}
\left(\Lambda-\Lambda_{c}\right) \xi & =\Lambda_{c} a \xi^{2}+\Lambda_{c} b \xi^{3}+\ldots \\
& -\Lambda_{c} \alpha \bar{\xi}_{2}-\left(\Lambda-\Lambda_{c}\right) \beta \bar{\xi}_{2}+O\left(\xi \bar{\xi}_{2}\right)
\end{aligned}
$$

Expressions for the postbuckling coefficients " $a$ " and " $b$ " and the imperfection forms factors " $\alpha$ " and " $\beta$ " are derived in References [28,29]. If the limit point is close to the bifurcation point, then the maximum load $\Lambda_{S}$ that the structure can carry prior to buckling can be evaluated from Eq. (14) by maximizing $\Lambda$ with respect to $\xi$. For cases where the first postbuckling coefficient " $a$ " is zero, this analysis vields the modified Koiter formula [29]

$$
\left(1-\rho_{s}\right)^{3 / 2}=\frac{3}{2} \sqrt{-3 \alpha^{2} b}\left[1-\frac{\beta}{\alpha}\left(1-\rho_{s}\right)\right]\left|\bar{\xi}_{2}\right|(15)
$$

where $\rho_{s}=\Lambda_{s} / \Lambda_{c}$.

Notice that, if the second postbuckling coefficient "b" is positive, Eq. (15) has no real solutions. Thus the buckling load of the specified shell-loading combination is not sensitive to small asymmetric initial imperfections of the shape given by Eq. (13). If, however, the second postbuckling coefficient " $b$ " is negative, the equilibrium load $\Lambda$ decreases following buckling and the buckling load of the real structure $\Lambda_{s}$ is sensitive to the asymmetric initial imperfection specified by Eq. (13).

\section{Level-1 Analysis of Asymmetric} Imperfection

For the isotropic shell under investigation, as can be seen from the partial results listed in Table 2, there are many identical eigenvalues. Hence, strictly speaking, the proposed form of the perturbation expansion given by Eqs. (14) is not applicable, since the nonlinear interaction between the many nearly simultaneous eigenmodes is not accounted for. Thus the following results, where one considers the eigenfunctions corresponding to certain critical eigenvalues chosen one at the 
time, can at best give an indication as to the severity of the expected imperfection sensitivity.

Initially, we assume a long wave asymmetric imperfection affine to one of the critical buckling modes of the perfect Caltech isotropic shell A-8, computed by the Level-1 computational module AXBIF (see also Table 3)

$$
\bar{W}=h \bar{\xi}_{2} \sin \pi \frac{x}{L} \cos 9 \frac{y}{R}
$$

Next using the Level-1 computational module BFACT to carry out the initial postbuckling analysis yields the following results

$$
\begin{aligned}
& \lambda_{c}=\lambda_{c}^{m}=1.00004(m=1, n=9) \\
& b=-0.010636 \quad \alpha=\beta=1.0
\end{aligned}
$$

Substituting these values into Eq. (15), one can plot the degrading effect of an asymmetric imperfection of the shape given by Eq. (16) as a function of its amplitude $\bar{\xi}_{2}$. As can be seen from Fig. 11 an initial imperfection amplitude equal to the wall thickness of the shell $\left(\bar{\xi}_{2}=10\right)$ generates a "knockdown factor" of $\lambda_{s}=0.679$, resulting in the following low buckling load

$$
\begin{aligned}
N_{S}= & \lambda_{s} N_{c \ell}= \\
& 0.679(-49.51517)=-33.621 \mathrm{lb} / \mathrm{in}
\end{aligned}
$$

Notice that the imperfection form factors " $\alpha$ " and " $\beta$ " are identical equal to 1.0 because BFACT uses membrane prebuckling to calculate the necessary first and second order fields and the assumed asymmetric imperfection shape of Eq. (16) is affine to the buckling mode. If, however, one assumes the following short-wave asymmetric imperfection

$$
\bar{W}=h \bar{\xi}_{2} \sin 17 \pi \frac{x}{L} \cos 27 \frac{y}{R}
$$

which lies also on the Koiter circle, then the initial postbuckling analysis, done once again by the Level-1 computational module BFACT, yields the following results

$$
\begin{aligned}
& \lambda_{c}=\lambda_{c}^{m}=1.00025(m=17, n=27) \\
& b=-220.84 \quad \alpha=\beta=1.0
\end{aligned}
$$

Using these values in Eq. (15) one obtains Fig. 12. As can be seen, an initial imperfection amplitude equal to the shell wall thickness $\left(\xi_{2}=1.0\right)$ this time generates a "knockdown factor" of $\lambda_{s}=0.0249$, resulting in an unrealistically low buckling load of

$$
\begin{aligned}
& N_{s}=\lambda_{s} N_{c \ell}= \\
& \quad 0.0249(-49.51517)=-1.233 \mathrm{lb} / \mathrm{in}
\end{aligned}
$$

Here one obviously cannot rely on the prediction of the Level-1 computational module BFACT, which uses membrane prebuckling analysis. In such a situation, as has been shown by Hutchinson and Frauenthal [30], one must repeat the postbuckling analysis using a rigorous prebuckling solution which includes the nonlinear effects due to the edge restraints.

\section{Level-2 Analysis of Asymmetric} Imperfection

To investigate the effects of edge-constraint and/or different boundary conditions on the imperfection sensitivity of the critical buckling load of the Caltech isotropic shell A-8 one has to switch to the Level-2 module ANILISA [15] and run its postbuckling analysis option. In this module, as described earlier, the axisymmetric prebuckling state is represented by Eqs. (3), the buckling modes by Eqs. (4) and the postbuckling state by

$$
\begin{aligned}
& W^{(2)}=h\left[W_{\alpha}(x)+w_{\beta}(x) \cos n \theta\right] \\
& F^{(2)}=\frac{E R h^{2}}{c}\left[f_{\alpha}(x)+f_{\beta}(x) \cos 2 n \theta\right]
\end{aligned}
$$

where $\theta=y / R$. Details of the computational procedures used are reported in Refs. $[15,26]$.

Initially, let us assume that the specified asymmetric imperfection is given by Eq. (16)

$$
\bar{W}=h \bar{\xi}_{2} \sin \pi \frac{x}{L} \cos 9 \theta
$$

where $\theta=y / R$. Running ANILISA with rigorous prebuckling and SS-3 boundary conditions $\left(N_{X}=-N_{0}, \quad v=w=M_{X}=0\right)$ yields the following results

$$
\begin{aligned}
& \lambda_{c}=\lambda_{c}^{n l}=0.934990(n=9) \\
& b=-0.016202 ; \alpha=0.20830 ; \beta=-0.38232
\end{aligned}
$$

Using Eq. (15) to plot the degrading effect of the asymmetric imperfection specified by Eq. (16) as a function of its amplitude $\bar{\xi}_{2}$ one 
obtains the results displayed in Fig. 13 as a solid line.

Here one must remember that Koiter's Sensitivity Theory is asymptotically exact, that is, it yields accurate predictions for sufficiently small imperfections, whereby what is sufficiently small may vary from case to case. Also, Eq. (15) was obtained by using the perturbation expansion given by Eq. (14), where terms of order $(\xi \vec{\xi})$ are neglected. As can be seen from the dotted curve plotted in Fig. 13, by using more advanced computational modules such as COLLAPSE [31], where a full nonlinear solution is used and terms up to and including order $\left(\xi \bar{\xi}^{2}\right)$ are kept, one obtains slightly lower predictions.

Notice that up to about $\bar{\xi}_{2}=0.1$ the asymptotic predictions from ANILISA and the nonlinear results of COLLAPSE agree very closely. Thus one can say that in this case the range of validity of the asymptotic solution is $0 \geq \bar{\xi}_{2} \leq 0.1$.

For an initial imperfection amplitude equal to the wall thickness of the shell $\left(\xi_{2}=1.0\right)$ the asymptotic solution yields a "knockdown factor" of $\lambda_{s}=0.806$, which corresponds to $a$ buckling load of

$$
\begin{aligned}
& N_{s}=\lambda_{s} N_{c \ell}= \\
& \quad 0.806(-49.51517)=-39.909 \mathrm{lb} / \mathrm{in}
\end{aligned}
$$

For the same initial imperfection amplitude of $\bar{\xi}_{2}=1.0$ the nonlinear solution using COLLAPSE yields a "knockdown factor" of $\lambda_{s}=0.68$, which corresponds to a buckling load of

$$
\begin{aligned}
& N_{s}=\lambda_{s} N_{C \ell}= \\
& \quad 0.68(-49.51517)=-33.670 \mathrm{lb} / \mathrm{in}
\end{aligned}
$$

Let us next consider the short wave asymmetric imperfection given by

$$
\bar{W}=h \bar{\xi}_{2} \sin 17 \pi \frac{x}{L} \cos 24 \theta
$$

where $\theta=y / R$. The rigorous asymptotic solution obtained by running ANILISA with SS3 boundary conditions $\left(N_{x}=-N_{0}\right.$, $v=w=M_{x}=0$ ) yields now the following results

$$
\lambda_{c}=\lambda_{c}^{n l}=0.844480(n=24)
$$

$$
b=-0.50329 ; \alpha=0.20113 ; \beta=-0.012022
$$

Using these values with Eq. (15) yiolds the solid curve plotted in Fig. 14. Rerunning the same case with the nonlinear module COLLAPSE yields the dotted curve plotted in Fig. 14.

Notice that in this case the asymptotic predictions from ANILISA and the nonlinear results from COLLAPSE agree closely up to about $\xi_{2}=0.03$. Thus the range of validity of the asymptotic solution is only $0 \geq \bar{\xi}_{2} \leq 0.03$.

For an initial imperfection amplitude of $\xi_{2}=1.0$ the asymptotic solution yields a "knockdown factor" of $\lambda_{\mathrm{s}}=0.400$, which corresponds to a buckling load of

$$
\begin{aligned}
& N_{s}=\lambda_{s} N_{c \ell}= \\
& \quad 0.400(-49.51517)=-19.806 \mathrm{lb} / \mathrm{in}
\end{aligned}
$$

For the same initial imperfection amplitude of $\bar{\xi}_{2}=1.0$ the nonlinear solution yields a "knockdown factor" of $\lambda_{s}=0.30$, which corresponds to a buckling load of

$$
\begin{aligned}
& N_{\mathrm{s}}=\lambda_{\mathrm{s}} N_{\mathrm{c} \ell}= \\
& \quad 0.30(-49.51517)=-14.855 \mathrm{lb} / \mathrm{in}
\end{aligned}
$$

For an initial imperfection amplitude equal to the wall thickness of the shell $\left(\bar{\xi}_{2}=1.0\right)$ the asymptotic solution yields a "knockdown factor" of $\lambda_{s}=0.806$, which corresponds to $a$ buckling load of

$$
\begin{aligned}
N_{s}= & \lambda_{s} N_{c \ell}= \\
& \quad 0.806(-49.51517)=-39.909 \mathrm{lb} / \mathrm{in}
\end{aligned}
$$

\section{MEASURED INITIAL IMPERFECTIONS}

In order to apply the theory of imperfection sensitivity with confidence, one must know the type of imperfections that occur in practice. In 1969 Arbocz and Babcock [1] published the results of buckling experiments where, for the first time, the actual initial imperfections and the prebuckling growth of the midsurface of electroplated isotropic shells were carefully measured and recorded by means of an automated scanning mechanism.

\section{Midsurface Initial Imperfections}

As can be seen from Fig. 15, the measured initial midsurface imperfections of shell A-8 [1] show a rather general distribution dominated 
by an $n=2$ mode. One can use the following double Fourier series

$$
\begin{aligned}
& \frac{\bar{W}}{h}=\sum_{i=1}^{7} \bar{W}_{i o} \operatorname{cosi\pi } \frac{x}{L}+\sum_{\ell=1}^{18} \bar{W}_{o \ell} \cos \ell \frac{y}{R}(20) \\
&+\sum_{\ell=1}^{19} \bar{W}_{0 \ell}^{\prime} \sin \ell \frac{y}{R}+\sum_{k, \ell=1}^{99} \sum_{k} \bar{W}_{k \ell} \cos k \pi \frac{x}{L} \cos \ell \frac{y}{R} \\
&+\sum_{k, \ell=1}^{115} \sum_{k} \bar{W}_{k \ell} \cos k \pi \frac{x}{L} \sin \ell \frac{y}{R}
\end{aligned}
$$

to represent the measured initial imperfections accurately, where Fourier coefficients with absolute values less than 0.001 are neglected.

A convenient measure of the size of the initial imperfections is their root-mean-square (RMS) value. By definition

$$
\Delta_{r m s}^{2}=\frac{1}{2 \pi R L} \int_{0}^{2 \pi R L} \int_{0}\left[\bar{W}(x, y]^{2} d x d y\right.
$$

Thus

$$
\begin{aligned}
\left(\frac{\Delta_{\mathrm{rms}}}{\mathrm{h}}\right)^{2}=\frac{1}{2} \sum_{\mathrm{i}} \overline{\mathrm{W}}_{\mathrm{io}}^{2} & +\frac{1}{4} \sum_{\mathrm{k}, \ell} \sum_{\mathrm{k} \ell}\left(\overline{\mathrm{W}}_{\mathrm{k} \ell}^{2}+\overline{\mathrm{W}}_{\mathrm{k} \ell}^{\prime 2}\right) \\
& =\Delta_{\text {axi }}^{2}+\Delta_{\text {asy }}^{2}
\end{aligned}
$$

For the A-8 shell the RMS values are

$$
\Delta_{\text {axi }}=0.5077 ; \Delta_{\text {asy }}=0.6882
$$

Using these measured RMS values with the previously discussed initial imperfection models yields the following buckling load predictions:

For the long wave

$$
\text { axisymmetric imperfection } \bar{W}=h \bar{\xi}_{1} \cos 2 \pi \frac{x}{L}
$$

if $\xi_{1}=\Delta_{\text {axi }}=-0.5077$ then from

$$
\begin{aligned}
& \text { MANILISA [27] } \quad \lambda_{c}^{n l}=0.824(n=9) \\
& P_{c}^{n l}=2 \pi R \lambda_{c}^{n l} N_{c \ell}=-1025.428^{l b s}
\end{aligned}
$$

For the short wave

$$
\text { axisymmetric imperfection } \bar{W}=h \bar{\xi}_{1} \cos 34 \pi \frac{x}{L}
$$

if $\xi_{1}=\Delta_{\text {axi }}=-0.5077$ then from
MANILISA [27] $\quad \lambda_{c}^{n !}=0.266(n=27)$

$P_{c}^{n l}=2 \pi R \lambda_{c}^{n l} N_{c \ell}=-331.024^{l b s}$

Turning now to the long wave asymmetric

$$
\text { imperfection } \bar{W}=h \bar{E}_{2} \sin \pi \frac{x}{L} \cos 9 \frac{y}{R}
$$

if $\bar{\xi}_{2}=\Delta_{\text {asy }}=0.6882$ then from

$$
\text { COLLAPSE [31] } \lambda_{c}^{n l}=0.740(n=9)
$$

$P_{c}^{n l}=2 \pi R \lambda_{c}^{n l} N_{c \ell}=-920.894^{l b s}$

For the short wave asymmetric

$$
\text { imperfection } \bar{W}=h \bar{\xi}_{2} \sin 17 \pi \frac{x}{L} \cos 24 \frac{y}{R}
$$

if $\bar{\xi}_{2}=\Delta_{\text {asy }}=0.6882$ then from

$$
\text { COLLAPSE [31] } \quad \lambda_{c}^{n !}=0.324(n=24)
$$

$P_{c}^{n l}=2 \pi R \lambda_{c}^{n l} N_{c \ell}=-403.202^{l b s}$

Comparing these buckling load predictions with the experimental buckling load of $P_{\exp }=-782.7^{\mathrm{bS}}$ it is clear that the long wave imperfection models yield an upper bound, whereas the short wave imperfection models yield a lower bound.

For a more accurate estimate of the buckling load one must use the measured initial imperfections in codes like STAGS $[20,21]$ to carry out 2-dimensional nonlinear collapse analysis. Employing a user written subroutine WIMP to input the double Fourier series of Eq. (20), the $161 \times 181$ STAGS model yielded with SS-3 boundary condition $\left(N_{x}=-N_{0}, v=w=M_{x}=0\right)$ a collapse load of $P_{S}=-900.103^{\text {lbs }}$, whereas the same model with $\mathrm{C}-4$ boundary condition $\left(u=u_{0}\right.$, $v=w=w, x=0$ ) yielded a collapse load of $P_{s}=-976.467^{\mathrm{lbs}}$. Both of these values are significantly higher than the experimental buckling load of $P_{\text {exp }}=-782.7^{\text {lbs }}$.

\section{Boundary Initial Imperfections}

In References $[32,33]$ it was shown that boundary imperfections can have a significant degrading effect on the buckling loads of axially compressed cylindrical shells. The flatness of the end ring, attached to the loadcell of the Caltech test set-up shown in Fig. 16, was measured about 20 years after it was 
used to test shell A-B. As can be seen in Fig. 17 there is a very large amplitude waviness of the end support, with maximum deviations of about \pm 3 wall thicknesses.

The measured boundary imperfections are decomposed in a 1-dimensional Fourier series

$u_{0}=\bar{u}_{b}(y)=$

$\bar{\xi}_{b} h\left\{\frac{1}{2} a_{o}+\sum_{n=1}\left(a_{n} \cos n \frac{y}{R}+b_{n} \sin n \frac{y}{R}\right)\right\}$

This series then is used in STAGS [20] to model the effect of boundary initial imperfections using a modified $\mathrm{C}-4$ boundary condition

$$
u=\bar{u}_{b}(y), v=w=w, x=0
$$

and taking advantage of the dual loading systems provided by the program.

Because the grooves of the end rings were filled with liquid Cerrolow at the time shell A-B was installed in the test set-up, it is to be expected that after cooling off the hardened liquid metal has filled-in all the gaps. The effect of this unknown end support was modeled by varying the amplitude of the boundary imperfection $\vec{\xi}_{b}$ between 0 and 0.1 . As can be seen from the results displayed in Fig. 18, including both the measured initial midsurface imperfections of Eq. (20) and the measured boundary imperfections of Eq. (23) with an amplitude of $\xi_{b}-0.035$, the calculated collapse load agrees well with the experimental buckling load.

\section{DISCUSSION OF THE RESULTS}

When comparing and analyzing the results obtained sotar it is important to keep in mind that all Level-1 and Level-2 solutions are based on approximate representations of the unknown functions. As pointed out in the previous sections Level- 1 solutions use a single term double Fourier series approximation to reduce the solution of the stability problem, formulated in terms of partial differential equations, to algebraic eigenvalue problems. The effect of edge restraint is neglected (one uses a membrane prebuckling solution) and the assumed field functions satisfy approximately SS-3 $\quad\left(\mathbf{N}_{\mathbf{X}}=-\mathbf{N}_{0}\right.$, $v=w=M_{X}=0$ ) boundary conditions.

Level-2 solutions eliminate the $y$ dependence by a truncated Fourier decomposition in the circumferential direction. The resulting system of nonlinear ordinary differential equations are solved numerically, whereby both the specified boundary conditions and the effect of edge restraint are rigorously satisfied. Thus by this approach the only approximation is that one represents the variation of the solution in the circumferential direction by a single harmonic with $n$ full waves, whereby an n-search is used to establish which wave number is the critical one. The Level-2 module ANILISA can also be used to investigate the effect of using different boundary conditions. In Table 5 , the results for four different boundary conditions are presented. As expected the fully clamped $\mathrm{C}_{4}$ boundary conditions has the highest critical buckling load. The increase in load carrying capacity with respect to the weaker SS3 boundary conditions is about the same as for an isotropic shell of similar characteristic dimensions (same L/R and $R / t$ ratios) of Ref. [34].

The Level-3 solutions are based either on a 2-dimensional finite difference or finite element formulation. In both cases, if one uses the appropriate meshes, one can obtain rigorous solutions where all nonlinear effects are properly accounted for. The only real problem with Level-3 type solutions is that for each problem one must establish the appropriate mesh size. Coarse meshes yield inaccurate solutions. What is coarse depends on the particular problem under investigation. Thus, for a general nonlinear solution a convergence study must always be carried out.

Using a hierarchical simulation platform such as DISDECO (Delft interactive Shell DEsign Code), where the analyst has at his disposal computational modules of different level of sophistication, such a convergence study can be carried out relatively quickly and accurately. In Table 5 a summary of the results obtained in this study is presented using normalized variables.

Table 5

Summary of buckling load calculations of the Caltech isotropic shell A-8

$$
\left(N_{c \ell}=-49.51517 \mathrm{lb} / \mathrm{in}\right)
$$

Periect shell analysis

Level-1 Membrane Prebuckling Repeated eigenvalues with high multiplicity

$$
\lambda_{c}^{m}=1.000 \rightarrow P_{c}=-1244.5^{l b s}
$$


Level-2 Nonlinear Prebuckling

$$
\begin{array}{rlrl}
\text { SS-3 } & \lambda_{c}^{n \mathrm{l}}=0.844480(n=24) \\
& \rightarrow & P_{c}=-1050.9^{\mathrm{lbs}} \\
\text { SS-4 } & \lambda_{c}^{n \mathrm{l}}=0.866974(n=25) \\
\rightarrow & P_{c}=-1078.9^{\mathrm{lbs}} \\
\text { C-3 } & \lambda_{c}^{n l}=0.910305(n=24) \\
& \rightarrow & P_{c}=-1132.8^{\mathrm{lbs}} \\
\text { C-4 } & \lambda_{c}^{n \mathrm{l}}=0.926871(n=25) \\
& \rightarrow & P_{c}=-1153.4^{\mathrm{lbs}}
\end{array}
$$

Level-3 SS-3 B.C.

$161 \times 181$ Model

$$
\begin{aligned}
\lambda_{c}^{n l}=0.833361(n=26) \\
\rightarrow P_{c}=-1037.1^{\mathrm{lbs}}
\end{aligned}
$$

$261 \times 261$ Model

$$
\begin{aligned}
\lambda_{c}^{n l}=0.844993(n & =24) \\
& \rightarrow P_{c}=-1051.6^{\text {lbs }}
\end{aligned}
$$

Imperfect Shell Analysis

Long wave axisymmetric imperfection -

$$
\xi_{1}=\Delta_{\text {axi }}=-0.5077
$$

Level-2 $\lambda_{c}^{n l}=0.824(n=9)$

$$
\rightarrow P_{C}=-1025.4^{l b s}
$$

Short wave axisymmetric imperfection -

$$
\xi_{1}=\Delta_{\text {axi }}=-0.5077
$$

Level-2 $\lambda_{c}^{n \prime}=0.266(n=27)$

$$
\rightarrow P_{c}=-331.0^{\text {lbs }}
$$

Long wave asymmetric imperfection -

$$
\bar{\xi}_{2}=\Delta_{\text {asy }}=0.6882
$$

Level-2 $\lambda_{c}^{n l}=0.740(n=9)$

$$
\rightarrow P_{S}=-920.9^{\text {lbs }}
$$

Short wave asymmetric imperfection -

$$
\bar{\xi}_{2}=\Delta_{\text {asy }}=0.6882
$$

Level-2 $\lambda_{c}^{n \prime}=0.324(n=24)$

$$
\rightarrow P_{s}=-403.2^{l b s}
$$

Measured midsurface initial imperfection ser Eq. (20)

$$
\text { Level-3 SS-3 B.C. } \rightarrow P_{S}=-900.1^{\text {Ibs }}
$$

Level-3 C-4 B.C. $\rightarrow P_{S}=-976.5^{\text {Ibs }}$

Measured midsurface + boundary $\left(\xi_{b}=0.035\right)$ initial imperfection - see Eqs. (20) and (23)

Level-3 Modified C-4 B.C.

(see Eq. (24)) $\rightarrow P_{s}=-780.0^{\text {lbs }}$

Experimental Buckling Load

$$
\rightarrow P_{\text {exp }}=-782.7^{\mathrm{lbs}}
$$

Notice that by including both the midsurface and the boundary initial imperfections one obtained a very good agreement indeed.

The study of the buckling behavior of the isotropic shell A-8 was undertaken with the objective of establishing a rational analysis procedure for incorporation of initial imperfections into the design of buckling critical structures. For a shell of revolution under axially symmetric loading there are the following four items necessary for the application of the proposed procedure:

a. Buckling analysis capability for shells of revolution under axially symmetric loading. (A computer code such as SRA [18] or BOSOR [35] will satisfy this requirement.)

b. Imperfection sensitivity analysis capability for shells of revolution under axially symmetric loading. (A computer code such as SRA [18] or STASOR [36] will satisfy this requirement.)

c. Geometrically nonlinear analysis capability for a shell of revolution, which can handle the asymmetry introduced by the general asymmetric initial imperfections. (The STAGS $[20,21]$ computer code will satisfy this requirement.)

d. A design imperfection.

At the present time this is the most elusive item.

The imperfection model introduced by Imbert [37], following an idea by Donnell and Wan [38], is one approach, but has yet to be extended to larger cylindrical shell structures and other shells of revolution.

\section{CONCLUSIONS}

By relying on a series of theoretical results of various degree of sophistication published in the literature, the hierarchical approach used in this paper has resulted in a series of buckling load predictions of increasing accuracy. It was shown that in order to be able to arrive at a reliable prediction of the critical buckling load 
and to make an estimate of its imperfection sensitivity which can be used with confidence, one must proceed step by step from simple to more complex models and solution procedures.

In particular one can state, that in order to predict the critical buckling load accurately and to make a reliable estimate of its imperfection sensitivity, the nonlinear effects caused by the edge restraint conditions must be included in the analysis. Any solution procedure which fails to account for these effects, should be suspect of having provided incorrect results.

The most approximate of the here described analyses, the Level-1 solutions which neglect the effects caused by the edge restraints, can still be used to great advantage to establish the approximate behavior of a shell subjected to the specified external loading. However, depending on the value of the prebuckling stiffness, resulting from the different types of wall constructions used, the solutions may be either conservative or nonconservative.

As can be seen from the results shown in Table 5, the buckling load of the isotropic shell A-8 is sensitive to all the initial imperfection shapes investigated. For a more specific prediction of the final collapse load, the final goal of a "High Fidelity Buckling Load Analysis", one has to carry out a refined Level3 analysis including measured values of all the significant generalized imperfections such as the traditional shell-wall imperfections, variations in the shell-end or loading surface geometry and especially for composite shells variations in the shell-wall thickness distribution. It has been shown in Ref. 33 that such an approach yields very good agreement between the predicted collapse load and the experimental buckling load.

\section{ACKNOWLEDGEMENT}

The research reported in this paper was supported in part by NASA Grant NAG 1-2129. This aid is gratefully acknowledged.

\section{REFERENCES}

1. Arbocz, J. and Babcock, C.D. Jr., "The Effect of General Imperfections on the Buckling of Cylindrical Shells," J. Appl. Mech., Vol. 36, 1969, pp. 28-38.

2. Arbocz, J., Starnes, J.H. and Nemeth, M.P., "A Hierarchical Approach to Buckling Load Calculations," Proceedings 40th AIAAASME/ASCE/AHS/ASC Structures, Structural Dynamics and Materials Conference, April 12-15, 1999, St. Louis, MO, pp. 289-299.
3. Anonymous, NASTRAN, The MacNealSchwendler Corporation, Los Angeles, California.

4. Anonymous, ABAQUS, Hibbitt, Karlsson \& Sorensen, Inc., Providence, Rhode Island.

5. Zienkiewicz, O.C., "The Finite Element Method," (The third, expanded and revised edition of The Finite Element Method in Engineering Science), McGraw-Hill Book Company (UK) Limited, London, 1977, p. 504.

6. Byskov, E., "Smooth Postbuckling Stresses by a Modified Finite Element Method," DCAMM Report No. 380, Technical University of Denmark, Lyngby, 1988.

7. Koiter, W.T., "On the Stability of Elastic Equilibrium," Ph.D. Thesis (in Dutch), THDelft, The Netherlands, H.J. Paris, Amsterdam, 1945, English translation NASA TTF-10, 1967, pp. 1-833.

8. Budiansky, B. and Hutchinson, J.W., "Dynamic Buckling of Imperfection Sensitive Structures," Proceedings 11th IUTAM Congress, Munich, 1964, Julius Springer Verlag, Berlin, 1966, pp. 636 651.

9. Hoff, N.J., "Buckling of Thin Shells," Proceedings of an Aerospace Symposium of Distinguished Lecturers in Honor of Theodore von Kármán on his 80th Anniversary, Institute of Aerospace Sciences, New York, 1961, pp. 1-42.

10. Stuhlman, C.E., De Luzio, A. and Almroth, B., "Influence of Stiffener Eccentricity and End Moment on Stability of Cylinders in Compression," AIAA Journal, Vol. 4, No. 5, May 1966, pp. 872-877.

11. Fischer, G., "Über den Einfluss der gelenkingen Lagerung auf die Stabilität dünnwandiger Kreiszylinderschalen unter Axiallast und Innendruck," Z.f. Flugwissenschaften, Vol. 11, 1963, pp. 111119.

12. Stein, M., The Influence of Prebuckling Deformations and Stresses on the Buckling of Perfect Cylinders," NASA TR190, February 1964.

13. Arbocz, J. and Hol, J.M.A.M., "Shell Stability Analysis in a Computer-Aided Engineering (CAE) Environment," Proceedings 34th AIAAASME/ASCE/ AHS/ASC Structures, Structural Dynamics and Materials Conference, April 19-22, 1993, La Jolla, California, pp. 300-314.

14. Arbocz, J., "The Effect of Initial Imperfections on Shell Stability - An Updated Review," Report LR-695, Delft University of Technology, Faculty of Aerospace Engineering, The Netherlands, September 1992. 
15. Arbocz, J. and Hol, J.M.A.M., "Koiter's Stability Theory in a Computer Aided Engineering (CAE) Environment," Int. J. Solids and Structures, Vol. 26, No. 9/10, pp. 945-973, 1990.

16. Booton, M., "Buckling of Imperfect Anisotropic Cylinders under Combined Loading," UTIAS Report No. 203, University of Toronto, 1976.

17. Von Kármán, T. and Biot, M.A. "Mathematical Methods in Engineering," McGraw-Hill Book Co., New York, London, 1940, p. 317.

18. Cohen, G.A., "Computer Analysis of Asymmetric Buckling of Ring-stiffened Orthotropic Shells of Revolution," AIAA Journal, Vol. 6, No. 1, January 1968, pp. 141-149.

19. Keller, H., "Numerical Methods of TwoPoint Boundary Value Problems," Blaisdell Publishing Co., Waltham, Mass., 1968.

20. Almroth, B.O., Brogan, F.A., Miller, E., Zele, F. and Peterson, H.T., "Collapse Analysis for Shells of General Shape; II. User's Manual for the STAGS-A Computer Code," Technical Report AFFDL-TR-71-8, Air Force Flight Dynamics Laboratory, Wright-Patterson Air Force Base, Ohio, March 1973.

21. Brogan, F.A., Rankin, C C. and Cabiness, H.D., "STags Access Routines-STAR Reference Manual," Report LMSC P032595, Version 2.0, Lockheed Palo Alto Research Laboratory, Palo Alto, California, June 1994.

22. Koiter, W.T., "The Effect of Axisymmetric Imperfections on the Buckling of Cylindrical Shells under Axial Compression," Koninkl. Ned. Akad. Wetenschap. Proc. B66, 1963, pp. 265-279.

23. Lorenz, R., "Achsensymmetrische Verzerrungen in dünnwandigen Hohlzylindern," Z..VDI, Vol. 52, 1908, pp. 1706-1713.

24. Timoshenko, S., "Einige Stabilitătsprobleme der Elastizitätstheorie," Z. Math. Phys., Vol. 58, 1910, pp. 337-385.

25. Southwell, R.V., "On the General Theory of Elastic Stability," Phil Trans. Roy. Soc. London, Series A, Vol. 213, 1914, pp. 187-244

26. Arbocz, J. and Hol, J.M.A.M., "ANILISA Computational Module for Koiter's Imperfection Sensitivity Theory, Report LR-582, Delft University of Technology, Faculty of Aerospace Engineering. The Netherlands, January 1989.

27. Romkes, A., "Stability and Imperfection Sensitivity of Anisotropic Cylindrical Shells under General Boundary Conditions," Memorandum M-809, Delft University of
Technology, Faculty of Aerospace Engineering, The Netherlands, July 1997.

28. Hutchinson, J.W. and Amazigo, J.C., "Imperfection Sensitivity of Eccentrically Stiffened Cylindrical Shells," AIAA Journal, Vol. 5, No. 3, March 1967, pp. 392-401.

29. Cohen, G.A., "Effect of a Nonlinear Prebuckling State on the Postbuckling Behavior and Imperfection Sensitivity of Elastic Structures," AIAA Joumal, Vol. 6, No. 8, August 1968, pp. 1616-1619.

30. Hutchinson, J.W. and Frauenthal, J.C., "Elastic Postbuckling Behaviour of Stiffened and Barreled Cylindrical Shells," J. Appl. Mech., Vol. 36, 1969, pp. 784790.

31. Arbocz, J., de Vries, J. and Hol, J.M.A.M., "On the Buckling of Imperfect Anisotropic Shells with Elastic Edge Supports under Combined Loading - Part I: Theory and Numerical Analysis", Memorandum M-849, Delft University of Technology, Faculty of Aerospace Engineering. The Netherlands, May 1997.

32. Arbocz, J., The Effect of Imperfect Boundary Conditions on the Collapse Behavior of Anisotropic Shells," Int. J. Solids Structures, Vol. 27, Numbers 46-47, 2000, pp. 6891-6915.

33. Hilburger, M.W. and Starnes, J.H. Jr., "Effects of Imperfections on the Buckling Response of Compression-Loaded Composite Shells," Proceedings 41st AIAA/ASME/ASCE/AHS/ASC Structures, Structural Dynamics and Materials Conference, 3-6 April 2000, Atlanta, Georgia, (Paper AIAA-2000-1382).

34. Almroth, B.O., "Influence of Edge Conditions on the Stability of Axially Compressed Cylindrical Shells", NASA CR-161, February 1965.

35. Bushnell, D., "Stress, Stability and Vibration of Complex Branched Shells of Revolution: Analysis and User's Manual for BOSOR-4," NASA CR-2116, 1972.

36. Weustink, A.P.D., "Stability Analysis of Anisotropic Shells of Revolution under Axisymmetric Load with General Boundary Conditions", Memorandum M-881, Delft University of Technology, Faculty of Aerospace Engineering, The Netherlands, November 2000.

37. Imbert, J., "The Effect of Imperfections on the Buckling of Cylindrical Shells," Aeronautical Engineer Thesis, California Institute of Technology, 1971.

38. Donnell, L.H. and Wan, C.C., "Effect of Imperfections on Buckling of Thin Cylinders and Columns under Axial Compression," J. App. Mech., Vol. 17, 1950, pp. 73-83. 


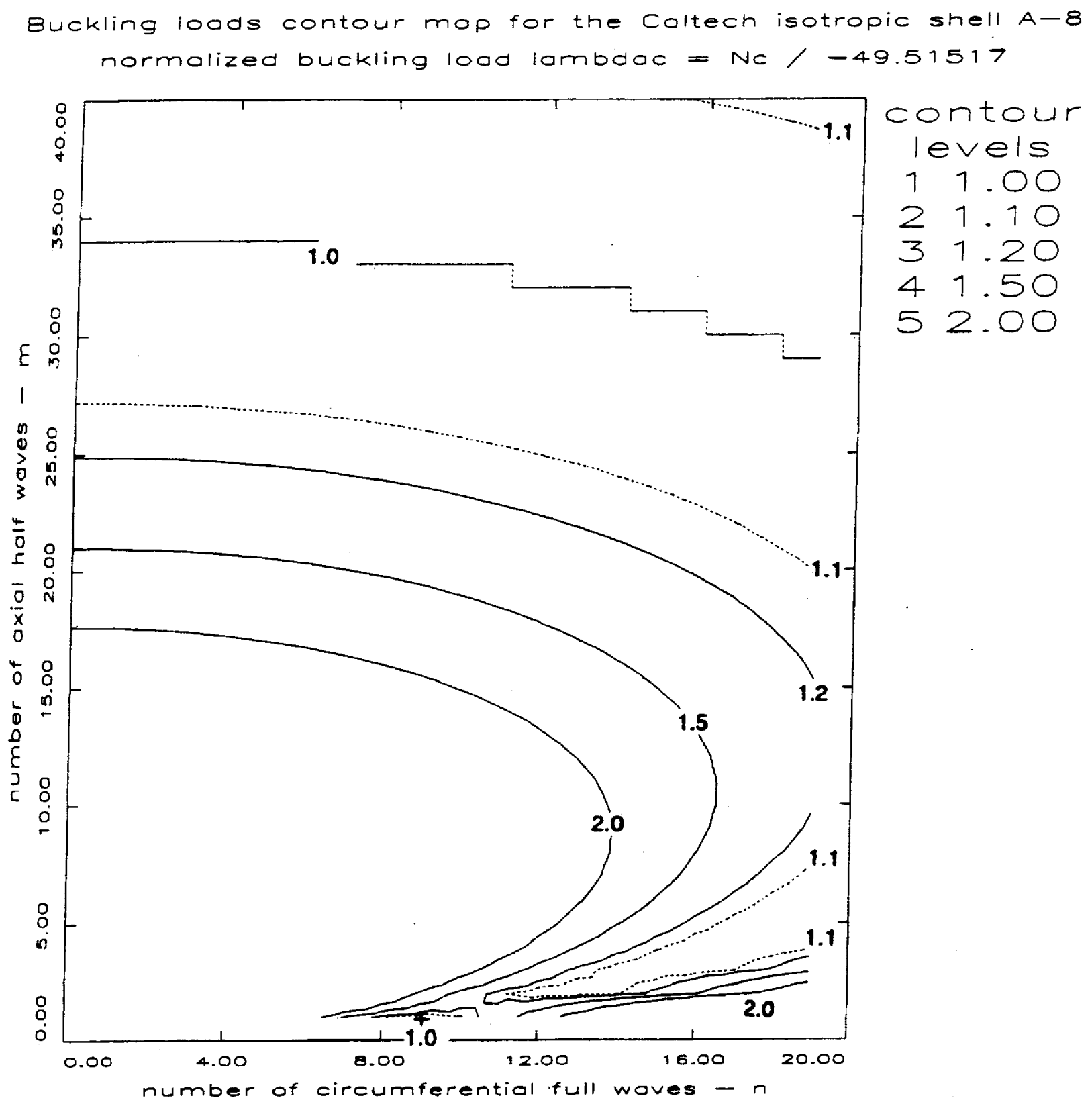

Fig. 1 Distribution of buckling loads based on Level-1 membrane prebuckling analysis - Caltech isotropic shell A-8. 


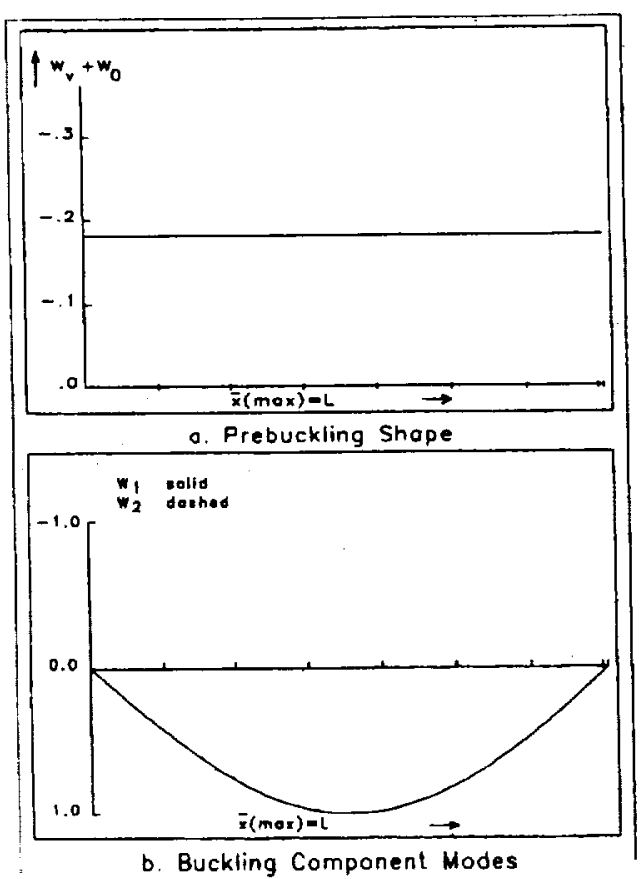

a. Membrane prebuckling, $n=9$

$\lambda_{c}^{m}=1.00006$

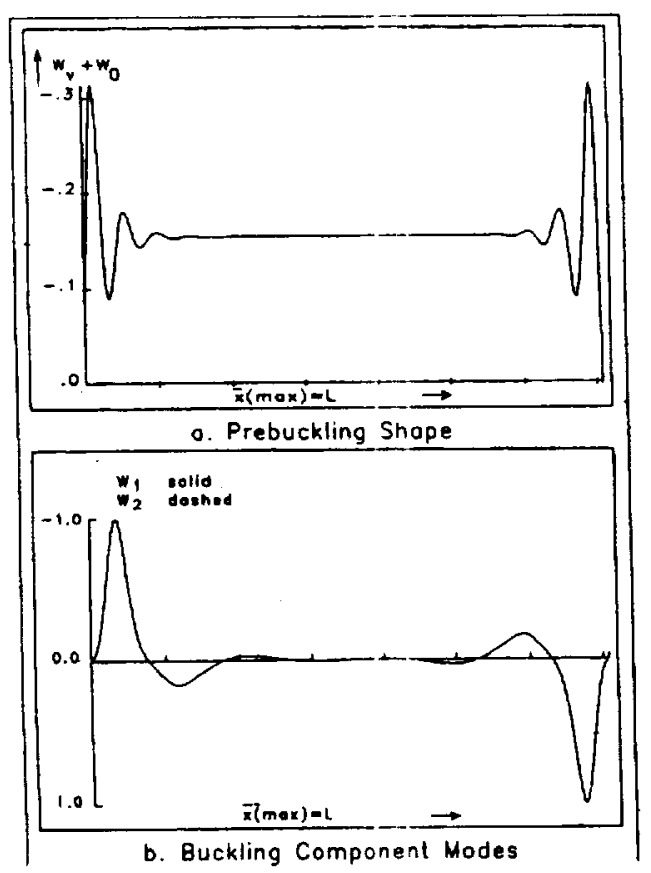

b. Nonlinear prebuckling, $n=24$

$\lambda_{c}^{n l}=0.844480$

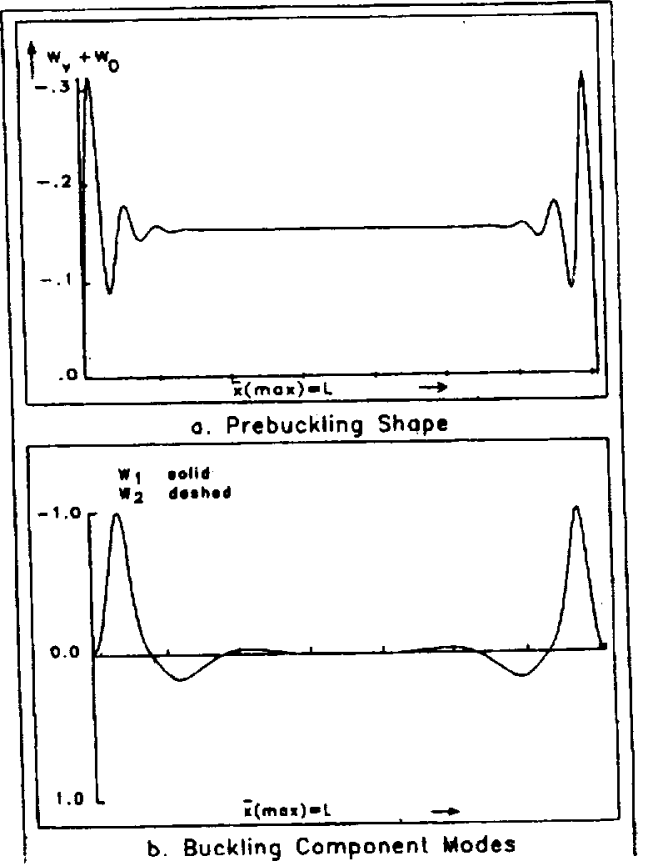

c. Nonlinear prebuckling, $n=24$ $\lambda_{c}^{\mathrm{nl}}=0.844481$

Fig. 2 Buckling modes of the axially compressed isotropic shell A-8

SS-3 B.C. $-N_{x}=-N_{0}, v=W=M_{x}=0: N_{c \ell}=-49.51517 \mathrm{lb} / i n$. 


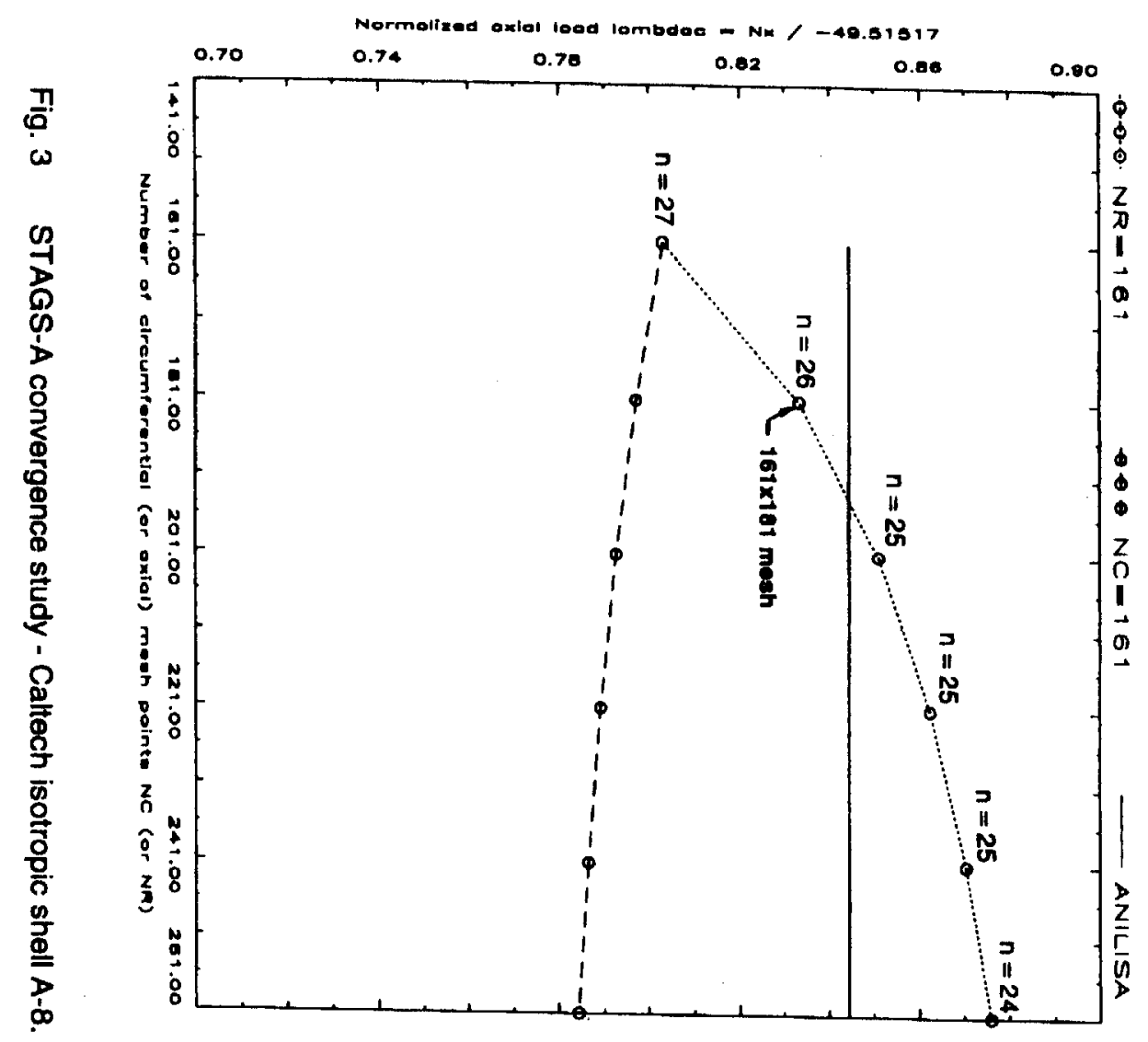




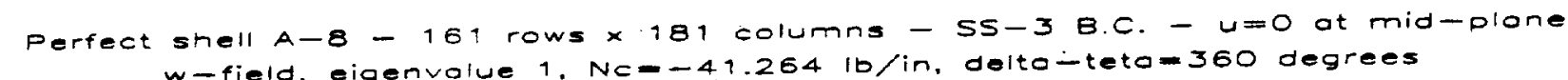

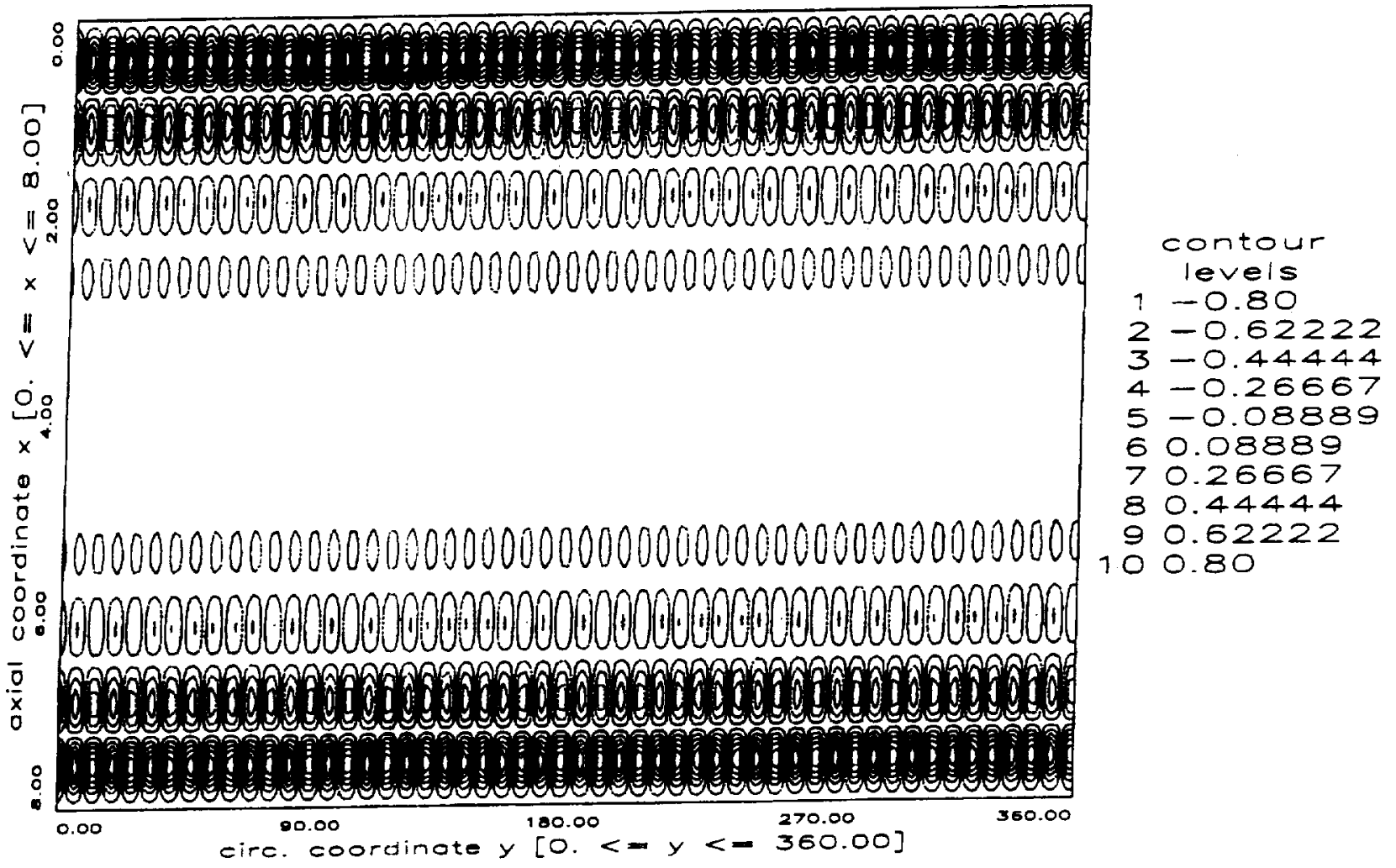

a. Contour-map of the critical (lowest) buckling mode

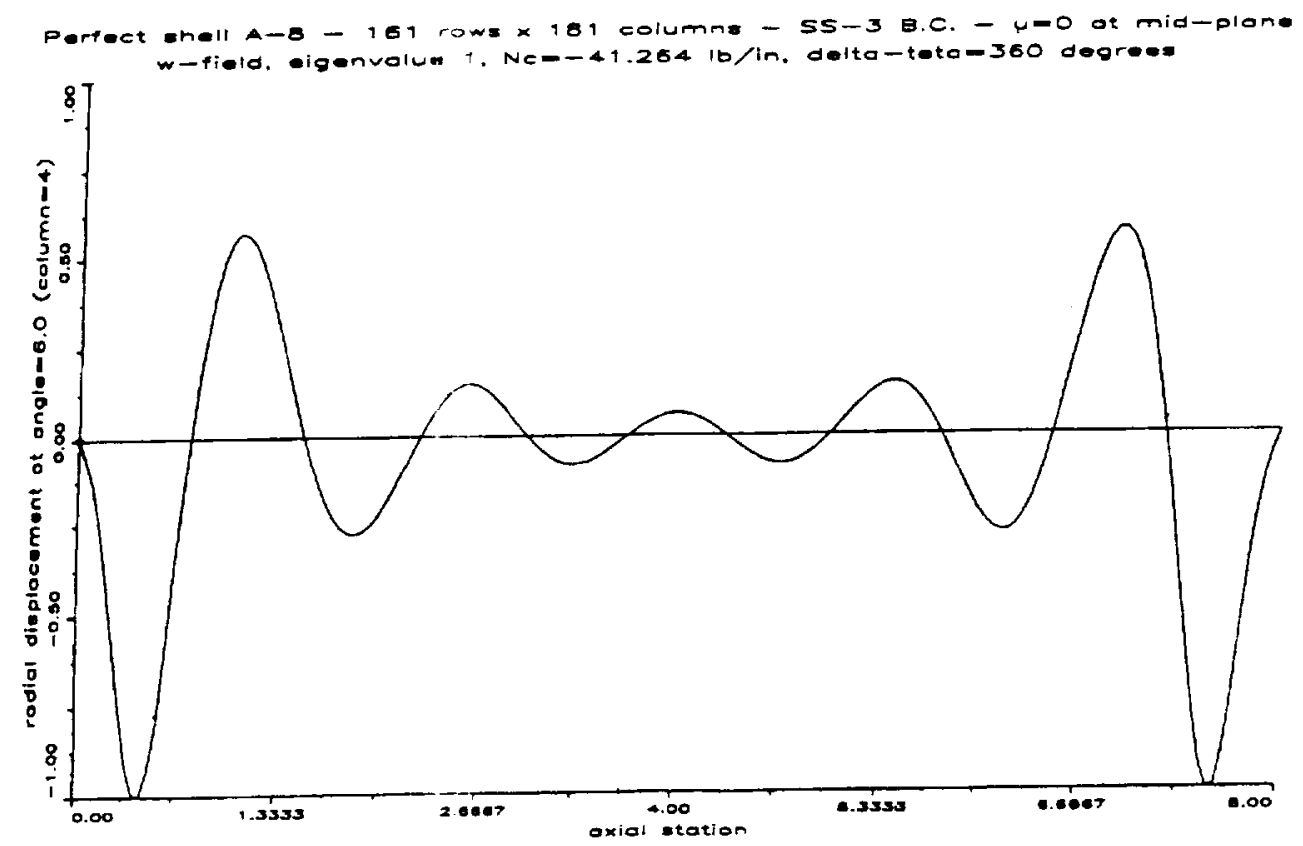

b. Axial trace of the critical (lowest) buckling mode

Fig. 4 Buckling mode of the axially compressed isotropic shell $A-8$ SS-3 B.C. $N_{x}=-N_{0}, v=W=M_{x}=0 ; N_{C \ell}=-49.51517 \mathrm{lb} / \mathrm{in}$; STAGS-A results. 


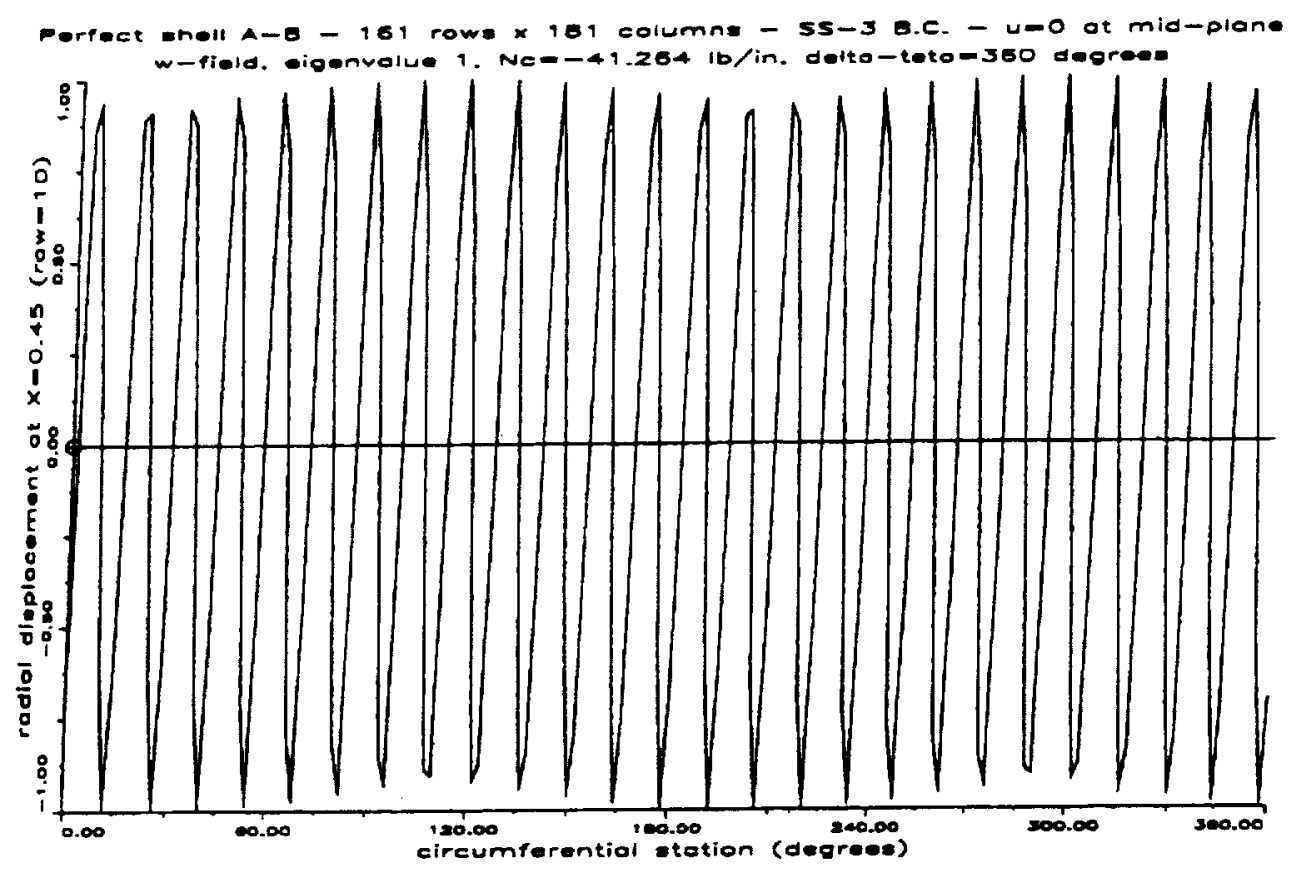

c. Circumferential trace of the critical (lowest) buckling mode

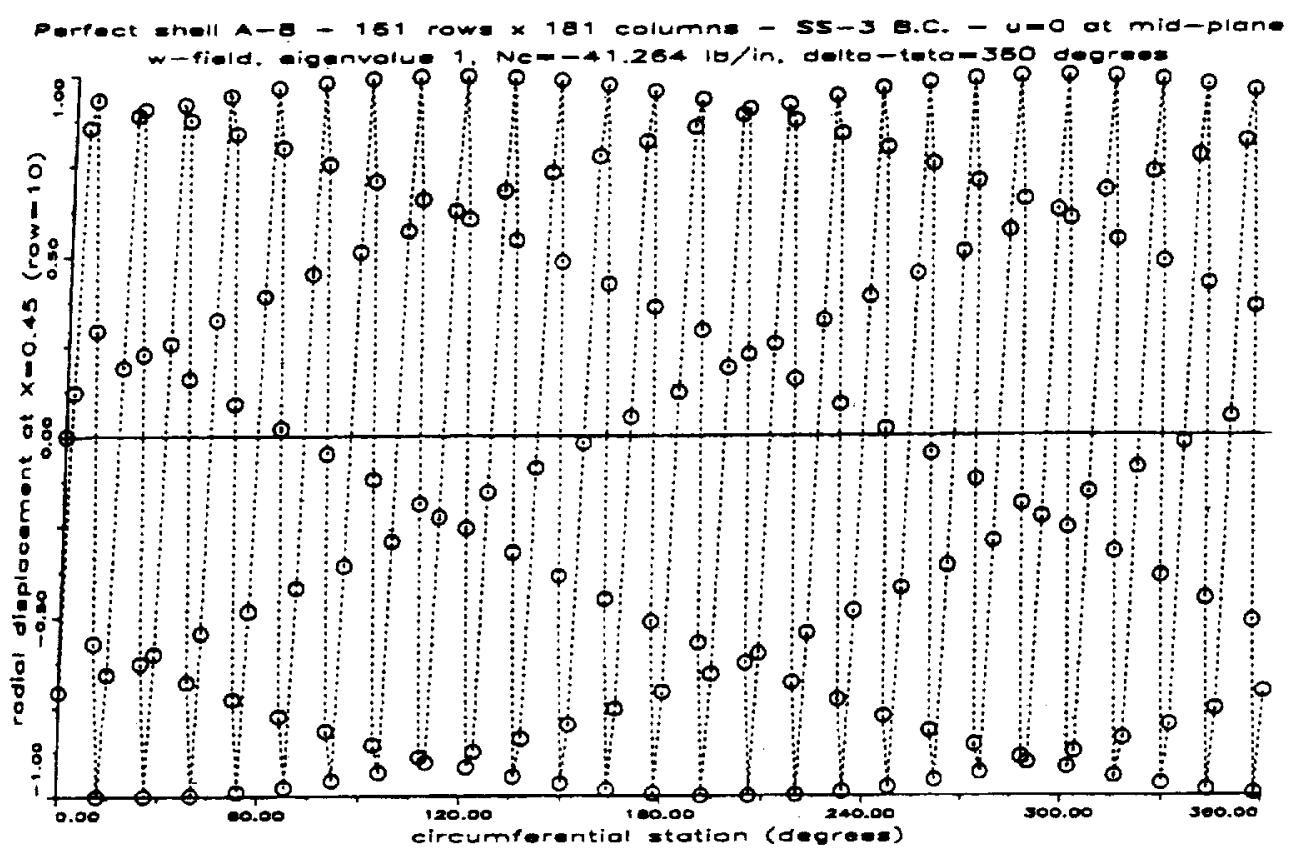

d. Circumferential trace of the critical (lowest) buckling mode

Fig. 5 Buckling mode of the axially compressed isotropic shell A-8

SS-3 B.C. $N_{x}=-N_{O}, v=W=M_{x}=0 ; N_{c \ell}=-49.51517$ lb/in; STAGS-A results. 


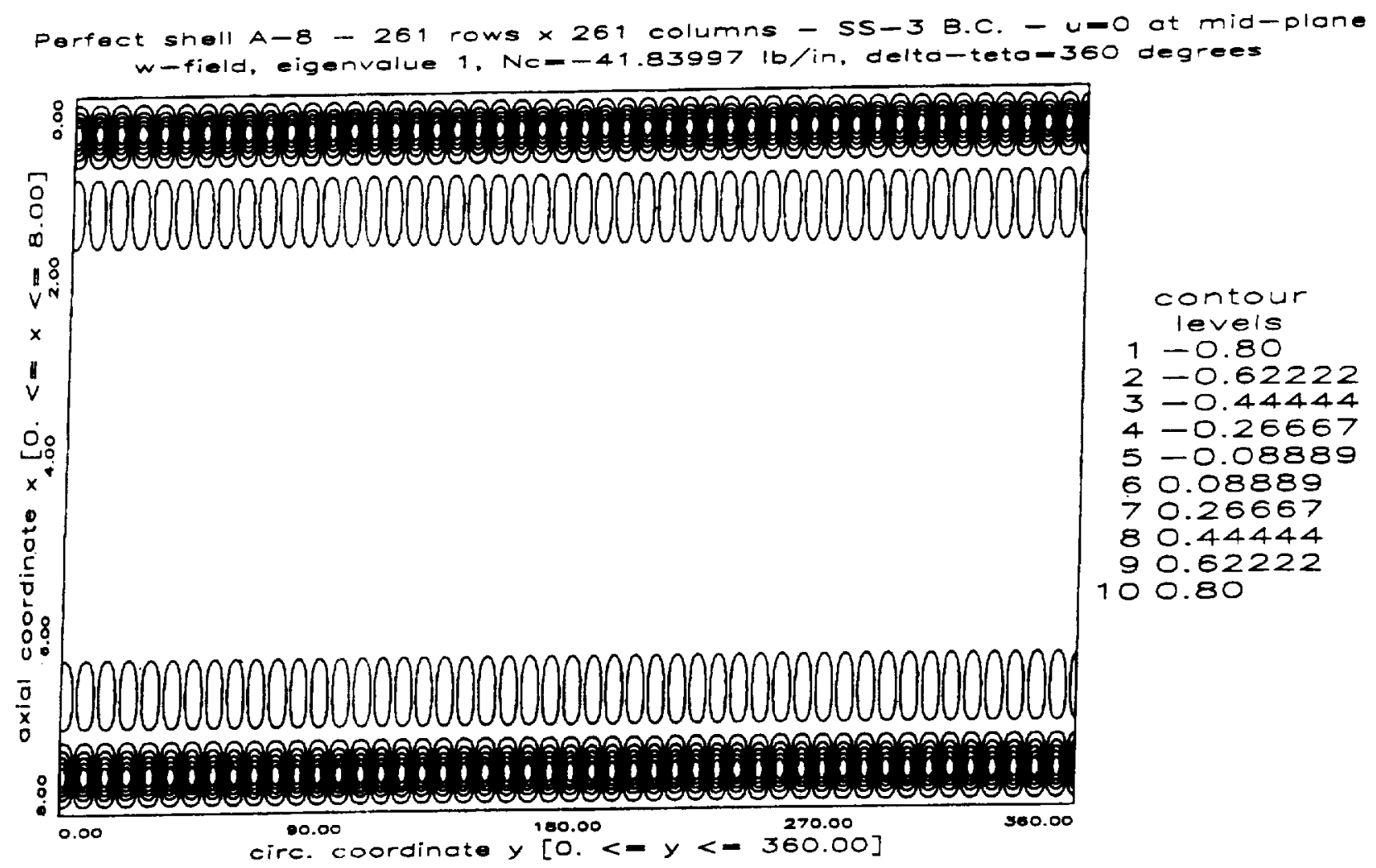

a. Contour-map of the critical (lowest) buckling mode

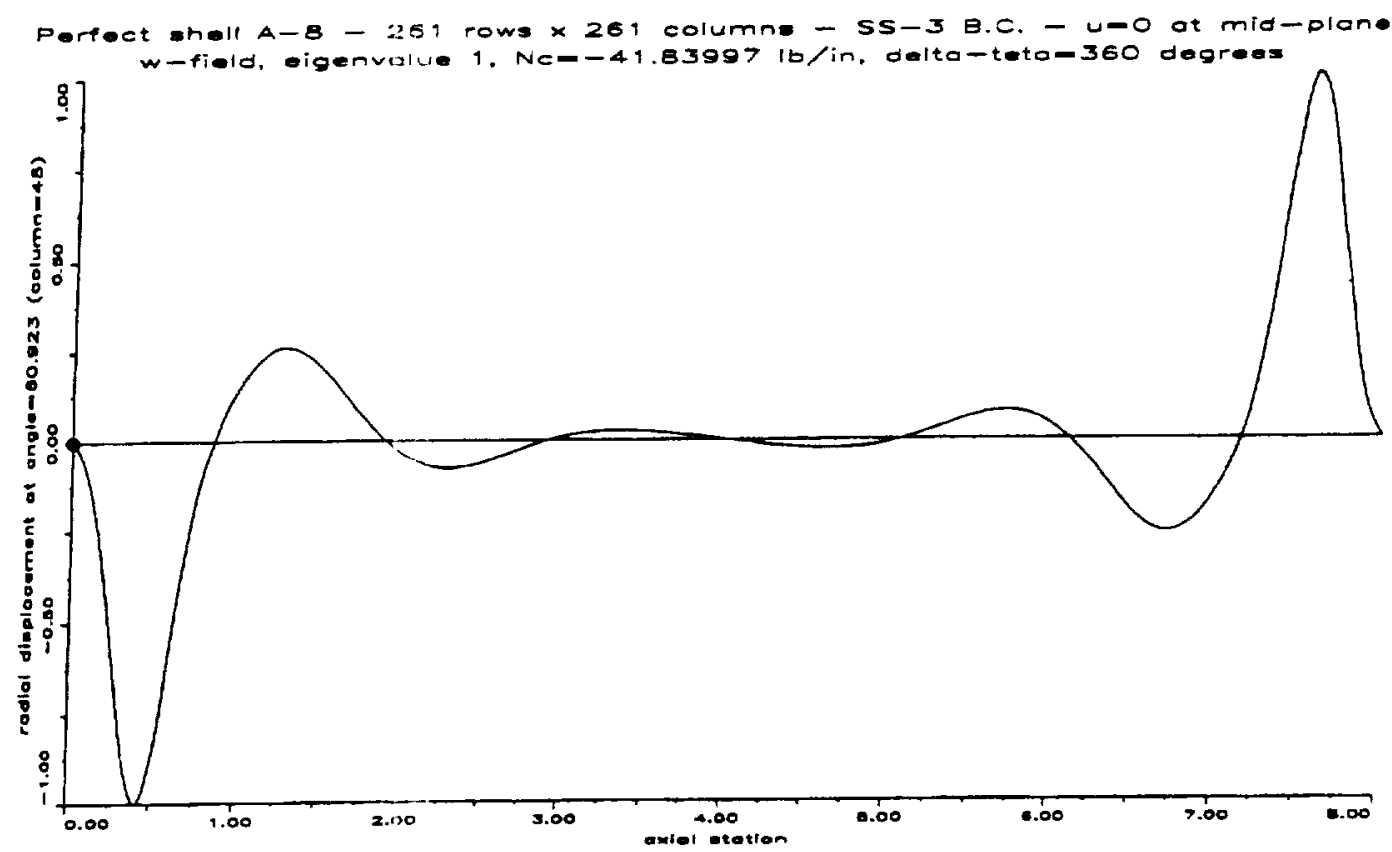

b. Axial trace of the critical (lowest) buckling mode

Fig. 6 Buckling mode of the axially compressed isotropic shell A-8

SS-3 B.C. $N_{X}=-N_{0}, v=W=M_{X}=0 ; N_{C \ell}=-49.51517$ lb/in; STAGS-A results. 


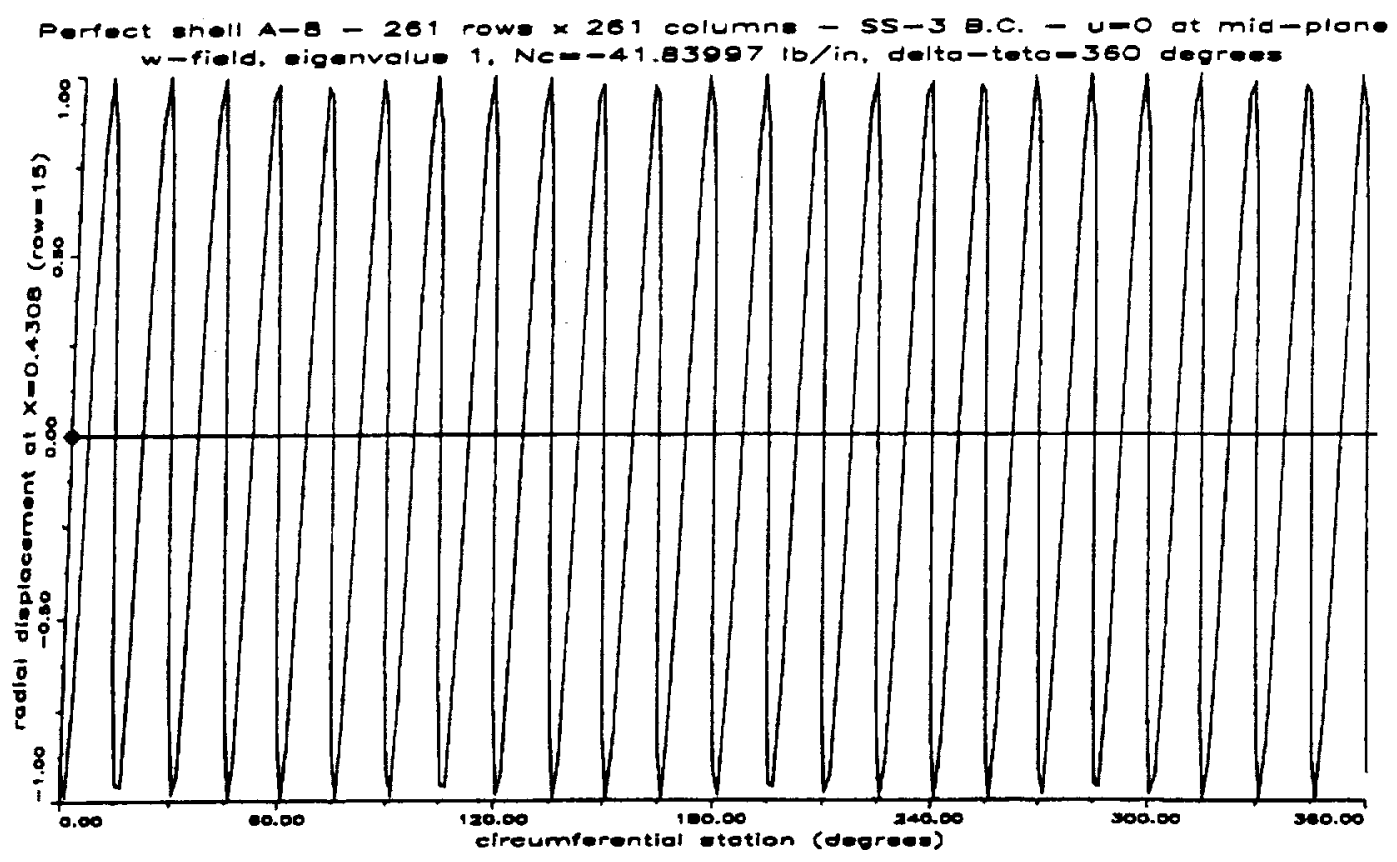

c. Circumferential trace of the critical (lowest) buckling mode

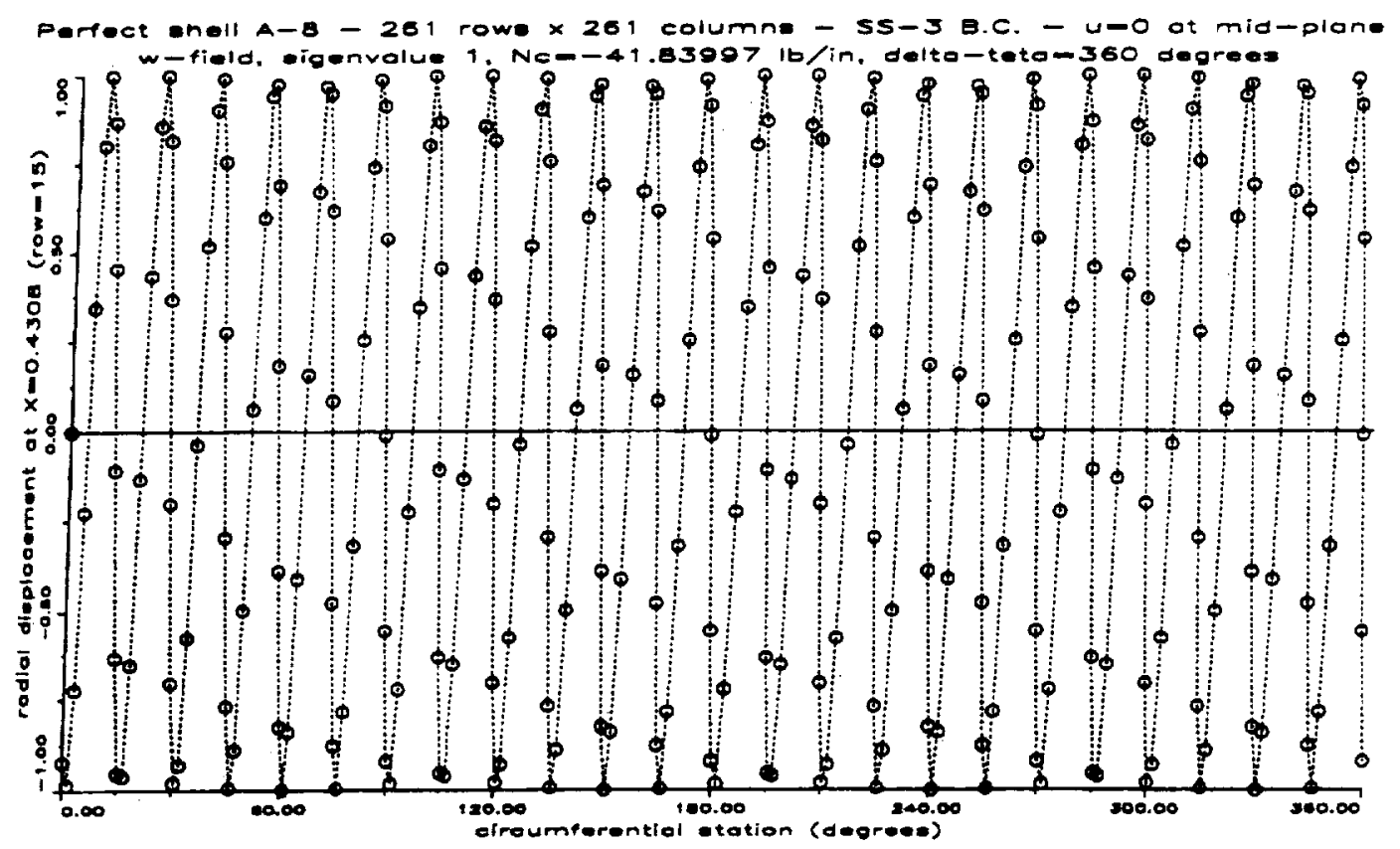

d. Circumferential trace of the critical (lowest) buckling mode

Fig. 7 Buckling mode of the axially compressed isotropic shell A-8

SS-3 B.C. $N_{x}=-N_{0}, v=W=M_{x}=0 ; N_{C \ell}=-49.51517$ lb/in; STAGS-A results. 


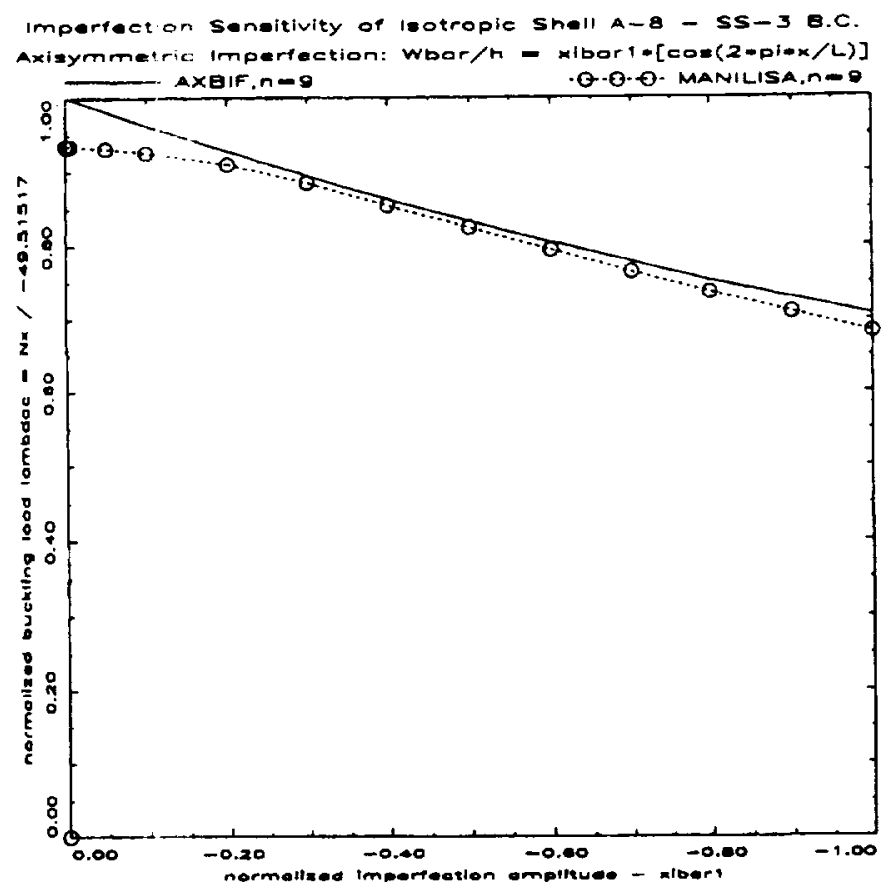

Fig. 8 Imperfection sensitivity for long wave axisymmetric imperfection under axial compression (SS-3 B.C.: $N_{X}=-N_{0}, v=W=M_{X}=0 ; N_{c \ell}=-49.51517$ lb/in).

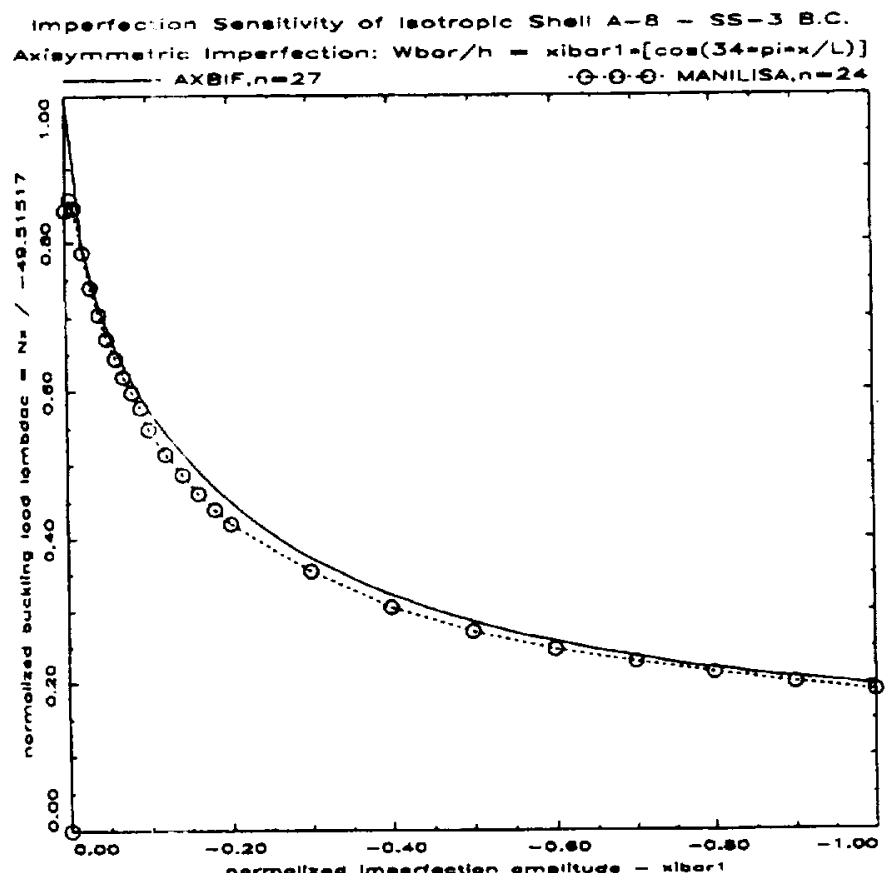

Fig. 9 Imperfection sensitivity for short wave axisymmetric imperfection under axial compression (SS-3 B.C.: $N_{x}=-N_{0}, v=W=M_{x}=0 ; N_{c \ell}=-49.51517 \mathrm{lb} / \mathrm{in}$ ). 


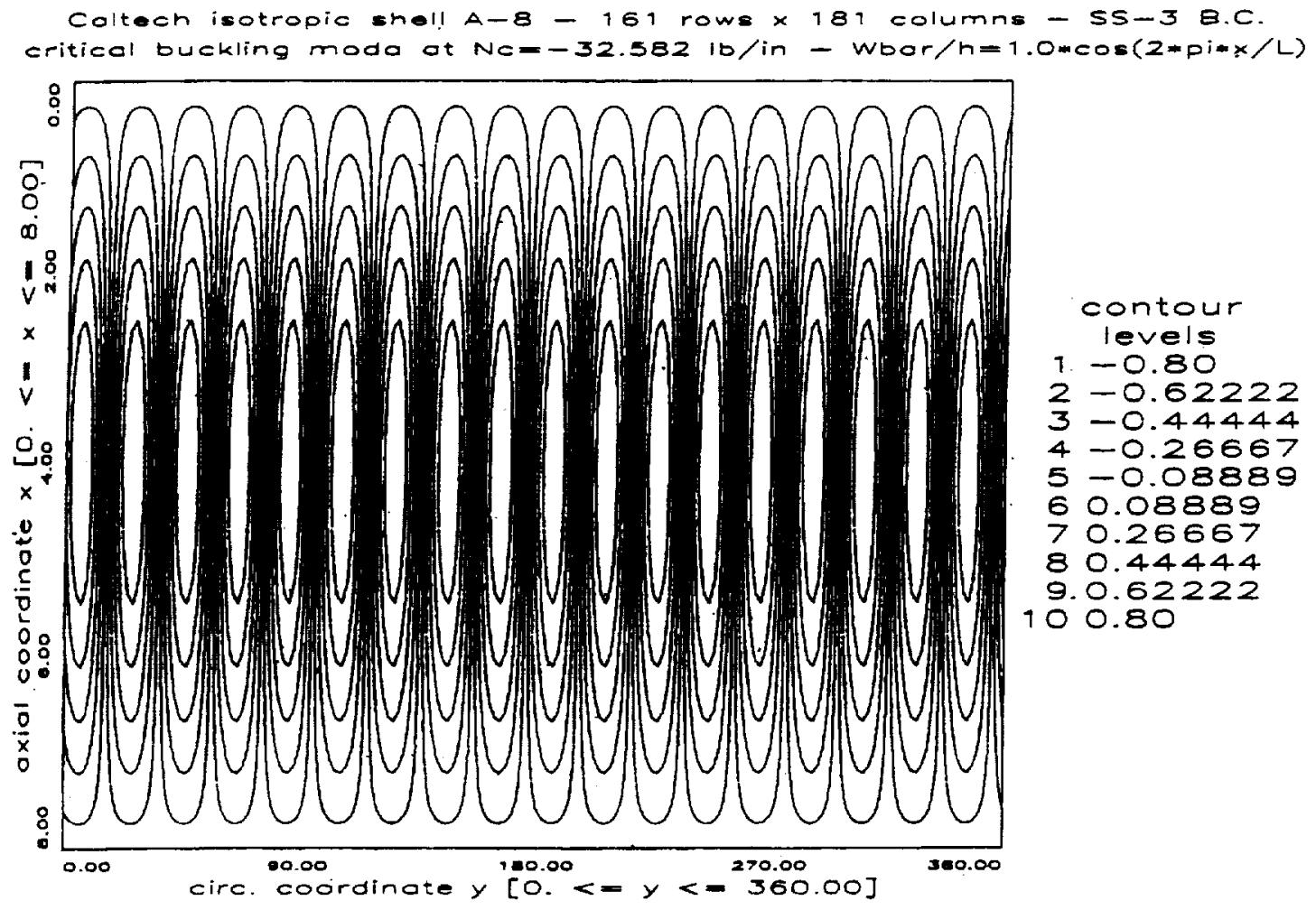

Fig. 10 Contour map of the critical buckling mode for axisymmetric imperfection. 


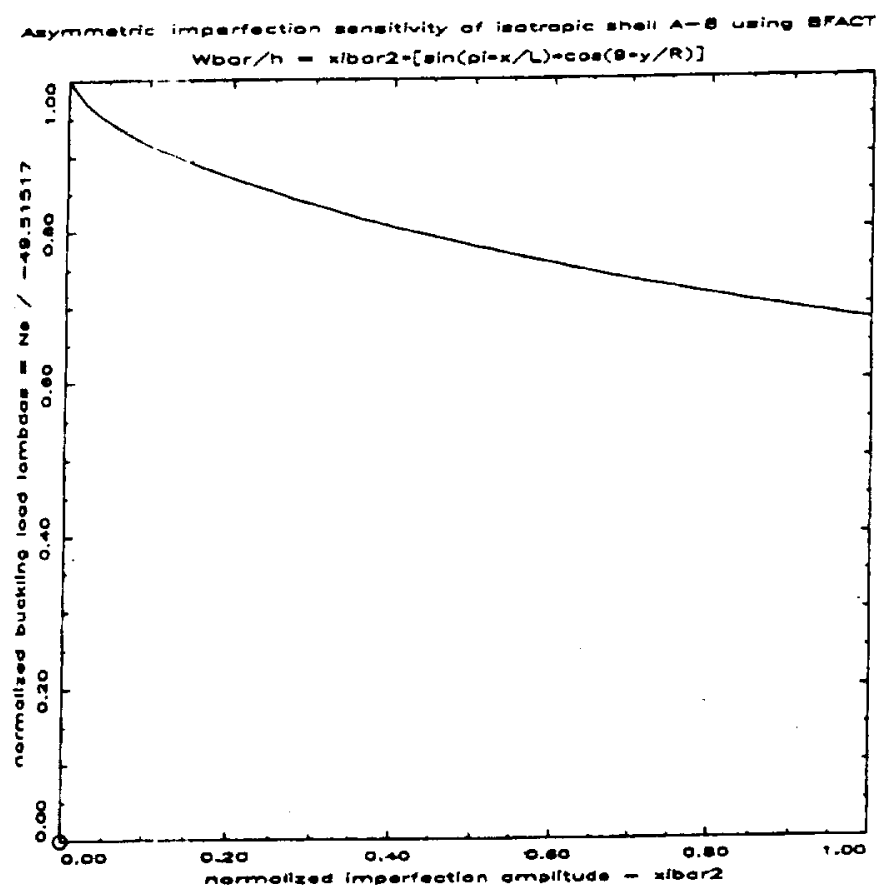

Fig. 11 Imperfection sensitivity for long wave asymmetric imperfection under axial compression using Level-1 routine BFACT $\left(\mathrm{N}_{\mathrm{C} \ell}=-49.51517 \mathrm{lb} / \mathrm{in}\right)$.

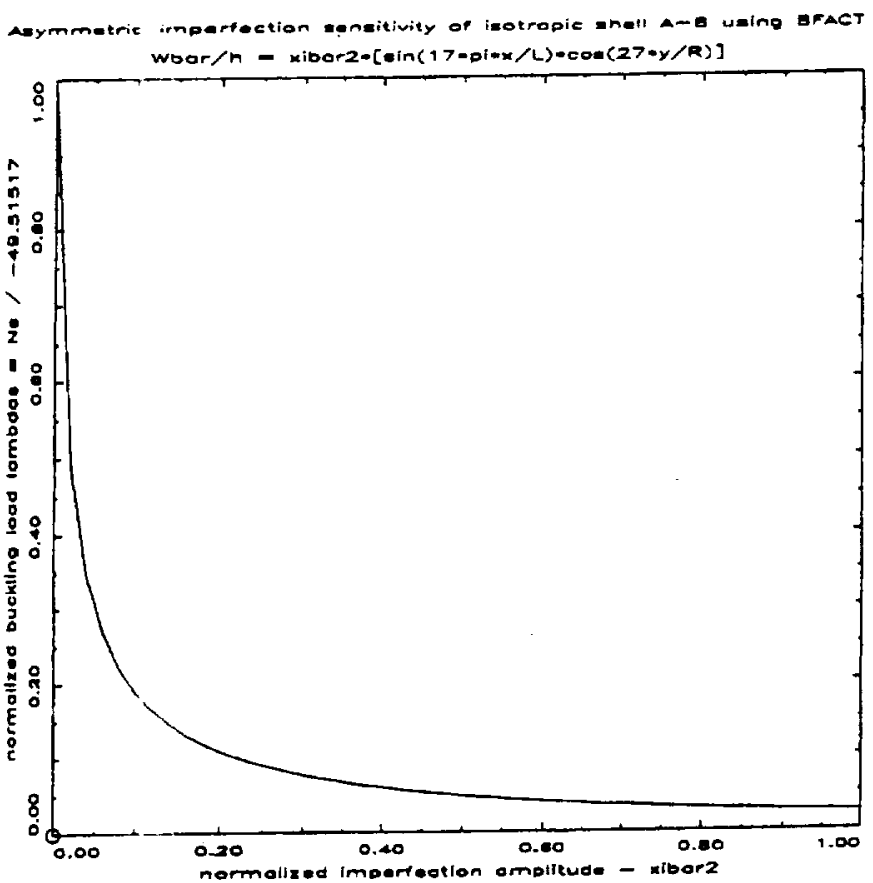

Fig. 12 Imperfection sensitivity for short wave asymmetric imperfection under axial compression using Level-1 routine BFACT $\left(N_{C \ell}=-49.51517 \mathrm{lb} / \mathrm{in}\right)$. 


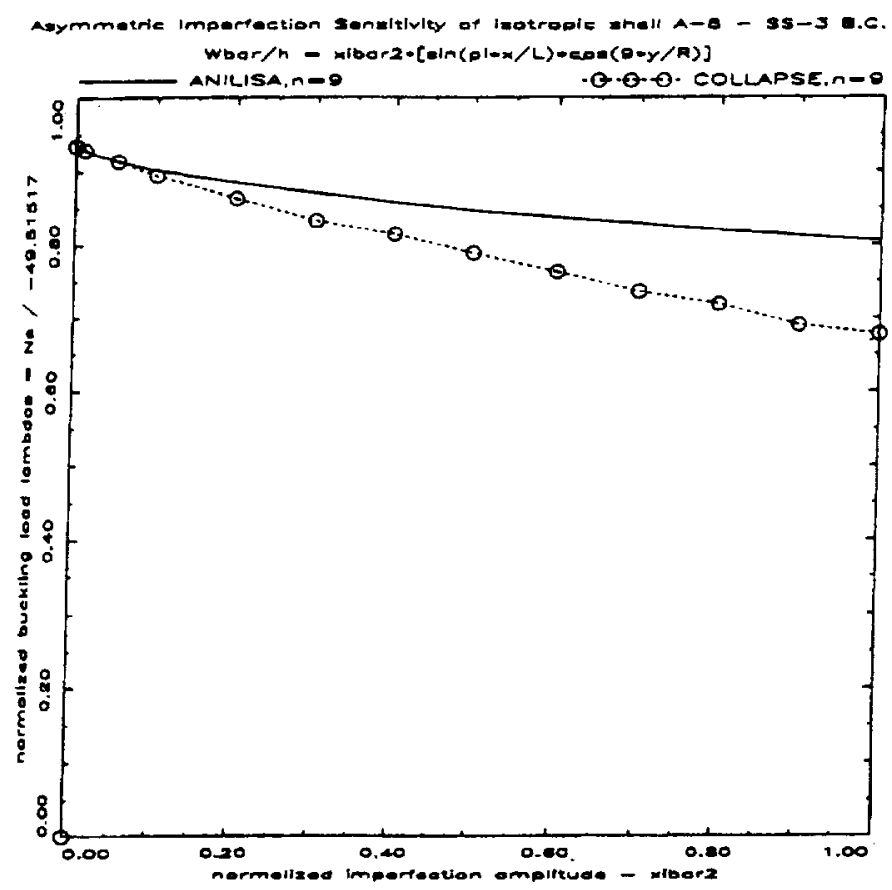

Fig. 13 Imperfection sensitivity for long wave asymmetric imperfection under axial compression (SS-3 B.C.: $N_{x}=-N_{0}, v=W=M_{X}=0 ; N_{c \ell}=-49.51517 \mathrm{lb} / \mathrm{in}$ ).

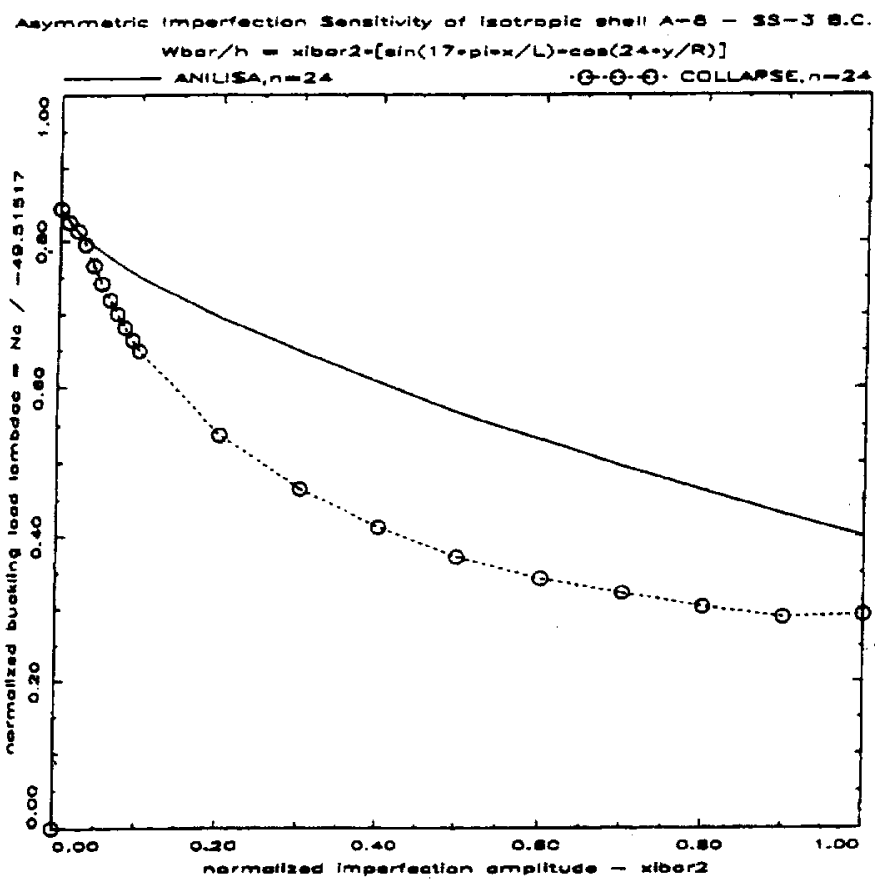

Fig. 14 Imperfection sensitivity for short wave asymmetric imperfection under axial compression (SS-3 B.C.: $N_{x}=-N_{0}, v=W=M_{x}=0 ; N_{c \ell}=-49.51517 \mathrm{lb} / \mathrm{in}$ ). 


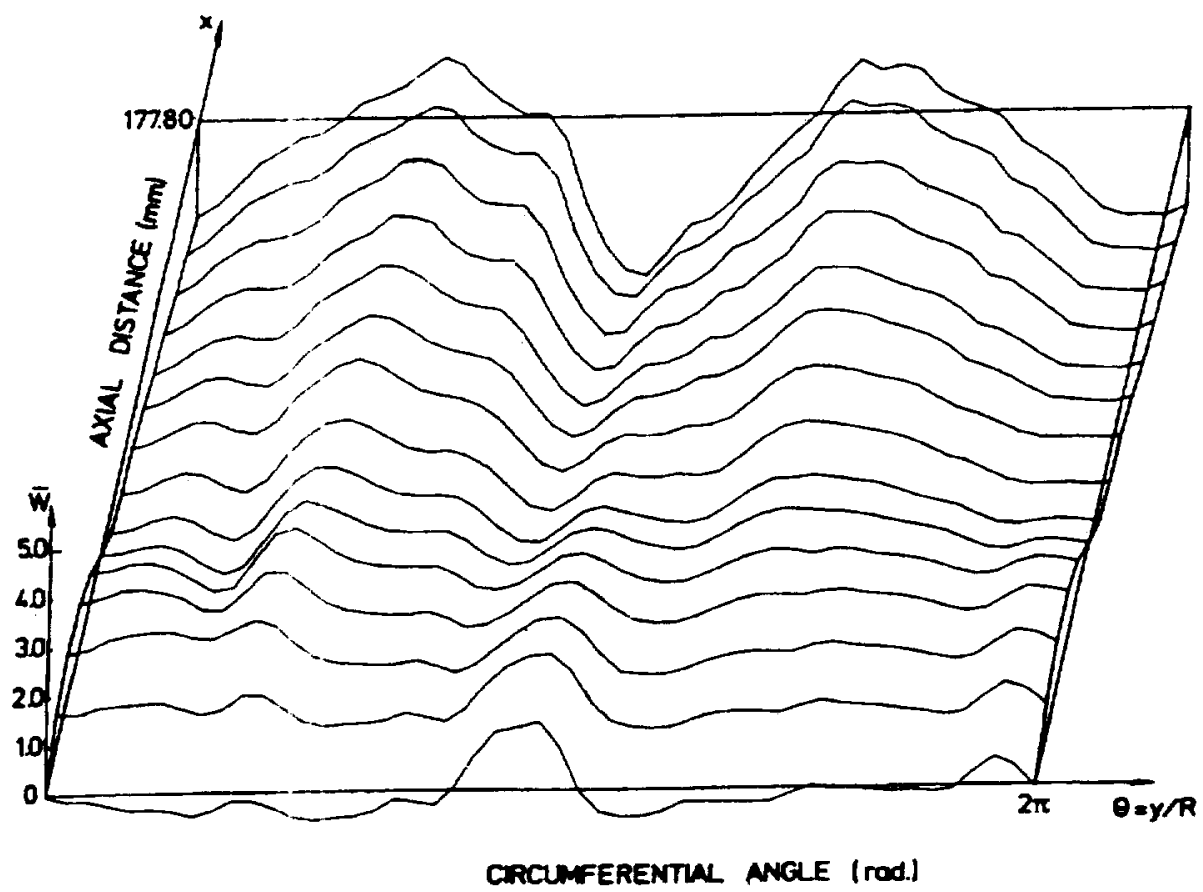

Fig. 15 Measured initial imperfections of Caltech isotropic shell A-8 [1].

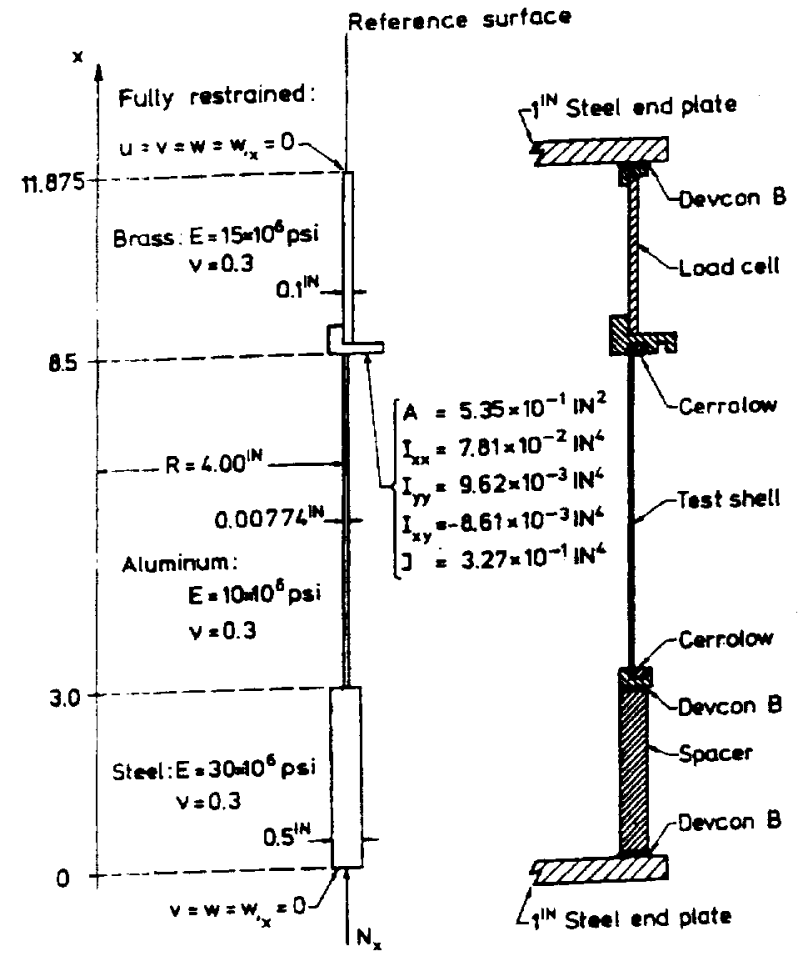

Fig. 16 Caltech test set-up and corresponding computer model. 


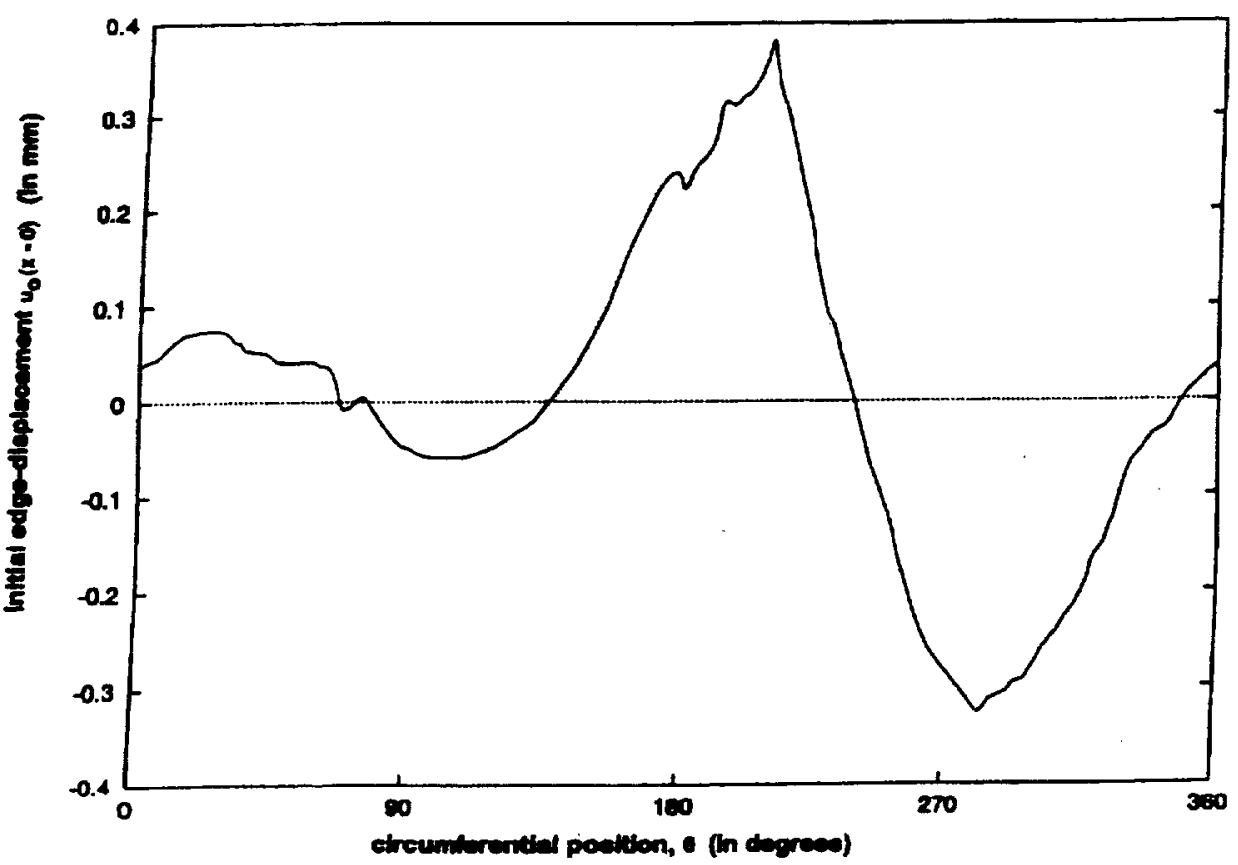

Fig. 17 Measured flatness of the Cahech load-cell end-ring.

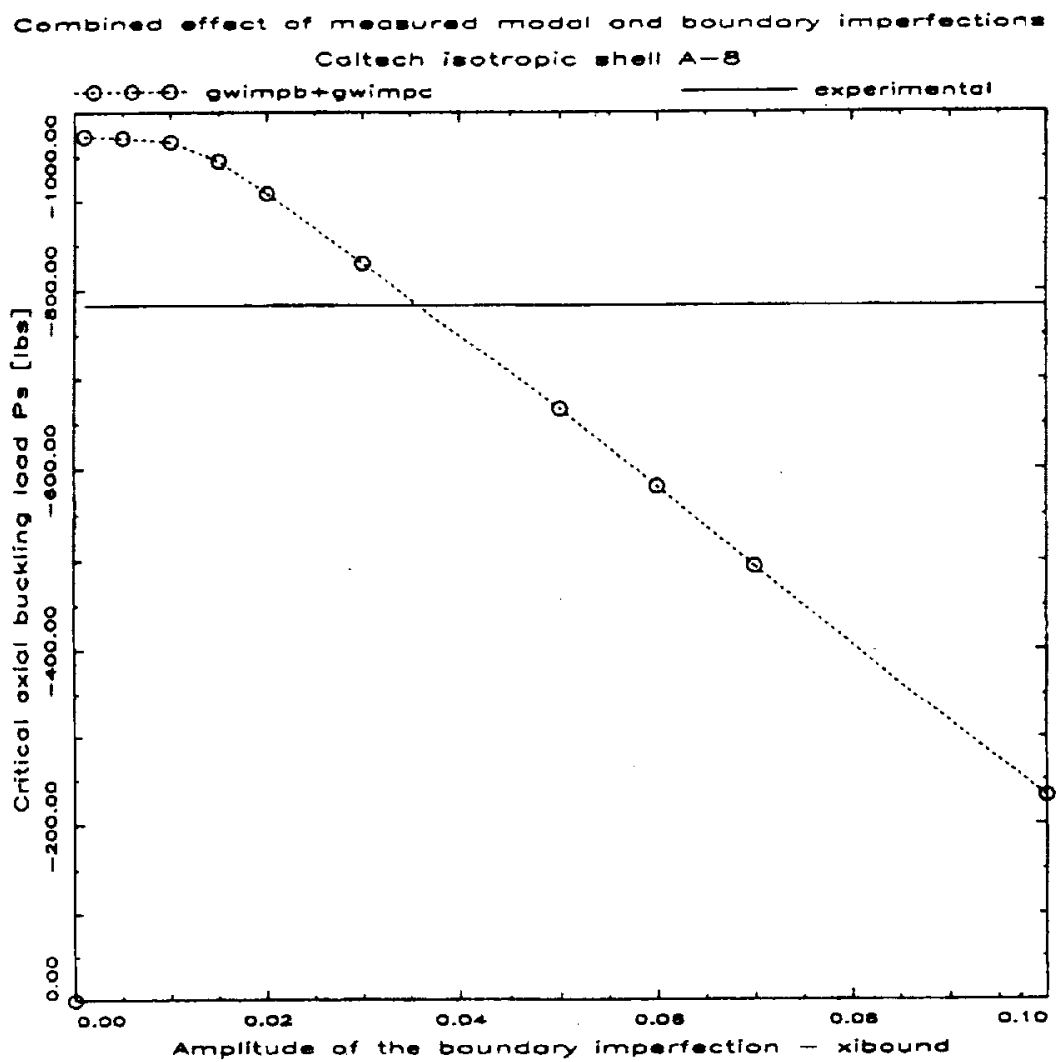

Fig. 18 Combined effect of measured modal and boundary imperfections. 